\title{
Channel noise in nerve membranes and lipid bilayers
}

\section{Journal Article}

\section{Author(s):}

Conti, F; Wanke, E.

Publication date:

1975

Permanent link:

https://doi.org/10.3929/ethz-b-000423011

\section{Rights / license:}

In Copyright - Non-Commercial Use Permitted

\section{Originally published in:}

Quarterly Reviews of Biophysics 8(4), https://doi.org/10.1017/S0033583500001967 
Quarterly Reviews of Biophysics 8, 4 (1975), pp. 45I-506

Printed in Great Britain

\section{Channel noise in nerve membranes and lipid bilayers}

\section{F. CONTI}

Laboratorio di Cibernetica e Biofisica, CNR, Camogli, Italy;

Laboratorium für Biochemie, ETH, Zürich, Switzerland

AND E. WANKE

Laboratorio di Cibernetica e Biofisica, CNR, Camogli, Italy

INTRODUCTION

THEORY

Fixed number of two-state channels 454

Fixed number of multistate channels 460

Fluctuations in channel number 463

Channel noise modulating intrachannel noise 464

EXPERIMENTAL TECHNIQUES

NoISE FROM NERVE MEMBRANES

Early studies ( $\mathrm{I} / f$ noise and burst noise) 468

Evidence for channel noise 470

Channel noise in voltage-clamped squid giant axons 471

Restrictions on possible channel models 478

NOISE FROM LIPID BILAYERS

Single channel conductance fluctuations 456

Noise in the presence of many channels $49 \mathrm{I}$

APPENDIX

Stochastic variables 493

Fluctuation-dissipation theorem 495

Statistical analysis of random signals 496

Modelling noise processes 498

Common sources of electrical noise 499

REFERENCES 


\section{INTRODUCTION}

The basic principles underlying fluctuation phenomena in thermodynamics have long been understood (for reviews see Kubo, I957; Kubo, Matsuo \& Kazuhiro 1973; Lax, 1960). Classical examples of how fluctuation analysis can provide an insight into the corpuscular nature of matter are the determination of Avogadro's number according to Einstein's theory of Brownian motion (see, e.g. Uhlenbeck \& Ornstein, 1930; Kac, 1947) and the evaluation of the electronic charge from the shot noise in vacuum tubes (see Van der Ziel, 1970). In most cases, however, fluctuations have been considered until recently as mere disturbances to the measurements of average values of macroscopic variables. To this aspect of the phenomenon the term 'noise' applies quite legitimately. Particularly in the field of electronics the minimization of noise for the purpose of optimizing measuring devices was the major task of fluctuation analysis. Also the initial studies of electrical noise in nerve membranes (Derksen, I965.; Derksen \& Verveen 1966; Verveen \& Derksen, I968) aimed merely at the understanding of, and accounting for, the disturbances produced by spontaneous fluctuations in the operation of the nervous system. The effects of membrane noise on thresholds, latencies, generator potentials, etc. were the main concern of these early investigations. For a review of this aspect of membrane noise see Lecar \& Nossal (1971).

The possibility of measuring the small electrical fluctuations occurring at the nerve membrane level led gradually to the realization that such phenomena could also provide a novel insight into the microscopic mechanisms of ionic conduction. The present review will be concerned exclusively with this latter aspect of membrane noise, either in nerves or in artificial lipid bilayers (BLM). The full application of noise techniques to BLM containing a large average number of extrinsic ionic channels, rather than the direct observation of single channel kinetics, has been made only in very recent studies (Neher \& Zingsheim, 1974; Zingsheim \& Neher, 1974; Kolb, Laüger \& Bamberg, 1975; Wanke, 1975). However, due to the precise characterization of these systems, the interpretation of the results is very clear and instructive. For this reason, and because of the relevance of the behaviour of these model membranes to the understanding of nerve membranes, fluctuation studies in BLM are also included in the review. Examination of the electrical fluctuations in postsynaptic membranes due to the chemically mediated 
open-close kinetics of ionic channels, constitutes the first and still the best example of the application of noise techniques to unravel the subtle mechanism of ionic conduction in biological membranes (Katz \& Miledi, 1970, 1972, I973; Anderson \& Stevens, 1973). The underlying phenomena and their theoretical interpretation, which led to important estimates of single channel parameters, are substantially the same as those postulated in nerve membranes or BLM containing pore forming substances. The acetylcholine receptor noise will not, however, be discussed here in any detail, since this subject is already treated exhaustively in the original works (Katz \& Miledi, I973; Anderson \& Stevens, 1973).

Although the present review is confined to the application of fluctuation analysis to study biological membranes, it is worth mentioning here that the exploitation of noise measurements is becoming common in many other fields of research. Chemical reactions (Feher \& Weissmann, 1973) and active transport (Segal, 1972; Van Driessche \& Borghgraef, 1975) are examples of such fields. The classical theoretical equivalence of fluctuation and relaxation analysis (see e.g. Kubo, 1957; Lax, 1960) is beginning to find wide experimental application. Due to the great improvement in the noise performance of commercially available solid-state devices, fluctuation analysis is today a valid alternative or a very powerful complement to the method of relaxation kinetics.

\section{THEORY}

We present in this section the theoretical characterization of that electrical noise which is expected to be found in membranes containing a discrete number of localized ionic pathways (channels), as a consequence of fluctuations in the conductance state and/or in the number of such channels. Throughout this article we shall use the term channel noise to indicate this type of fluctuation, which must be understood as clearly distinct from that which would occur if the number and the state of the channels were fixed. We shall see at the end of this section under what particular conditions these two types of noise may simply add to each other. When such conditions can be assumed to be valid, it is possible in principle to separate the two different noise contributions on the basis of their distinct spectral characteristics.

The term channel has here a general meaning, and can apply also to ionic pathways produced by the presence of mobile ionophores. We 
shall see, in fact, that the distinction between porous ionic pathways and ionophores does not imply qualitatively different types of noise. One can sometimes discriminate between the two mechanisms only on the basis of the absolute amplitude of the fluctuations to which they give rise.

We shall be mostly concerned with the case of statistically independent channels. This model is justified in general when the channels have a low surface density and can be assumed to be chemically stable molecular complexes, despite the fact that they might have a number of allowed internal conformational states. A large amount of evidence supports these assumptions insofar as the ionic channels of nerve membranes are concerned. However, when the channels originate from the formation of labile oligomeric complexes, as seems to be the case for BLM containing gramicidin $\mathrm{A}$ or alamethicin, the assumption of statistical independence cannot be made. An example of nonstatistically independent channels will be considered briefly when reviewing the results of gramicidin A induced noise in BLM.

For independent channels formal expressions have been derived (Chen \& Hill, 1973) which can be applied quite generally to any particular model of channel kinetics. We shall confine ourselves, however, only to those results which are easily expressed in a closed form and are sufficient to account for the presently available data on nerve fibres and lipid bilayers. We assume in this section that the reader has some familiarity with the basic concepts of stochastic processes and random variables. Otherwise the Appendix to this review should be read first. Most of the results that we report below have been extracted from the works of Stevens (1972), Hill \& Chen (1972a), and Chen \& Hill (1973).

\section{Fixed number of two-state channels}

One of the simplest types of channel noise arises when each channel out of a fixed number has only two statistically significant macroscopic states, and these states have different electrical conductances. The equally simple case of channels with only one state, but fluctuating in number, will be dealt with later. A macroscopic state of a channel is meant here as a state having an average life time which is much larger than that of any microscopic state compatible with it. It must also be stressed that, while a two-state channel will have in general two different conductance values, it is not generally true that channels with only two conductance states are two-state channels. Thus, the ionic channels in 
nerve membranes are usually described as having only two conductance states, but many different configurations with the same conductance. These channels are not two-state channels in the sense described above (Chen \& Hill, I973).

We first discuss the current fluctuations expected to occur when a fixed voltage, $E$, is applied across a membrane containing a single twostate channel. Let $\gamma$ be the highest channel conductance associated with state (I), let $\kappa \gamma(\kappa<I)$ be the conductance in state (o), and let $E_{m f}$ represent the membrane potential for zero current, which we assume for simplicity to be independent of the channel configuration. For a constant driving force, $V=E-E_{m f}$, the current flowing through the membrane, $I$, will fluctuate between the values $\kappa \gamma V$ and $\gamma V$, following the fluctuations of the channel between state (o) and state ( $\mathrm{I}$ ), respectively. Thus, the characterization of this latter stochastic process is necessary and sufficient for the description of our current fluctuations. The assumption that needs to be made, in order to achieve simple results, is that such process is markovian. This assumption, implying that the future development of the process depends solely on its present state and not on its past history, is indeed justifiable under very general conditions when the above definition of statistically significant channel states is born in mind (Lax, 1960). Our two-state markovian process is then described completely by the transition probabilities, $\Pi_{i, k}(t)(i, k=0, \mathrm{I})$ representing the probabilities of finding the channel in state $(k)$ at time $t$, provided it was in state $(i)$ at time zero. These are obtained as the solution of a simple system of first order linear differential equations (see e.g. Stevens, 1972):

$$
\left.\begin{array}{c}
\frac{\mathrm{d} \Pi_{i, k}}{\mathrm{~d} t}=\sum_{l=0}^{1} \Pi_{i, l} a_{l k}, \\
a_{01}=-a_{00}=\alpha, \quad a_{10}=-a_{11}=\beta,
\end{array}\right\}
$$

where the rate constants, $\alpha$ and $\beta$, are defined in such a way that $\alpha \Delta t$ and $\beta \Delta t$ give the transition probabilities, in the infinitesimal time interval $\Delta t$, from state (o) to state ( $\mathrm{I}$ ) or vice versa. The solution of equation ( $\mathrm{I}$ ) must satisfy the initial conditions

$$
\Pi_{i, k}=\delta_{i, k}, \quad\left\{\delta_{i, i}=\mathrm{I} ; \delta_{i, k}=0, k \neq i\right\}
$$

and is given by

$$
\Pi_{i, k}(t)=P_{k}+\left(\delta_{i, k}-P_{k}\right) \mathrm{e}^{-t / r},
$$


where

$$
P_{0}=\beta /(\alpha+\beta), \quad P_{1}=\alpha /(\alpha+\beta), \quad \tau=\mathrm{I} /(\alpha+\beta)
$$

$P_{0}$ and $P_{1}$ are the stationary (equilibrium) probabilities of finding the channel in state $(0)$ or in state $(\mathrm{I})$, respectively. The probability densities, $f_{T_{0}}(T)$ and $f_{T_{1}}(T)$, of the lifetimes $T_{0}$ and $T_{1}$, of state $(0)$ and state (I) respectively, are easily seen, from equations (2) and (3) to be exponentials

$$
\left.\begin{array}{rl}
f_{T_{0}}(T) \mathrm{d} T & =\left\{\lim _{\Delta t \rightarrow 0}(\mathrm{I}-\alpha \Delta t)^{T / \Delta t}\right\} \cdot \alpha \mathrm{d} T=\alpha \mathrm{e}^{-\alpha T} \mathrm{~d} T \\
f_{T_{1}}(T) \mathrm{d} t & =\left\{\lim _{\Delta t \rightarrow 0}(\mathrm{I}-\beta \Delta t)^{T / \Delta t}\right\} \cdot \beta \mathrm{d} T=\beta \mathrm{e}^{-\beta T} \mathrm{~d} T,
\end{array}\right\}
$$

with means

$$
\bar{T}_{0}=\mathrm{I} / \alpha, \quad \bar{T}_{1}=\mathrm{I} / \beta
$$

A typical time course of the current flowing through a single channel, undergoing the above stochastic process, is illustrated by the bottom trace of Fig. I. This signal was obtained by simulating the random process described by (I) with $\alpha \simeq \beta$ and assigning arbitrary values to the two current levels, $\kappa \gamma V$ and $\gamma V$. Indeed, as discussed later, similar single channel current fluctuations are actually observed in BLM containing EIM. For such systems there would be hardly any need of pursuing further our theoretical analysis as done below. The verification that the statistical distributions of the lifetimes $T_{0}$ and $T_{1}$ are simple exponentials provides in these cases a good test for the validity of the two-state model assumed above. Furthermore, the measurement of the two current levels and of the average lifetimes, $\bar{T}_{0}$ and $\bar{T}_{1}$ would yield all the channel parameters $(\gamma, \kappa, \alpha$ and $\beta)$ which can possibly be obtained at this level of experimentation. In most cases, however, particularly when dealing with natural membranes, the observation of isolated single channel processes is impossible. The observed current is the sum of the contributions from a large number of channels which independently and simultaneously undergo random fluctuations.

It will be clear from what follows that the introduction of the covariance, $\phi_{I}(t)$, is then essential. For the single channel case, $\phi_{I}(t)$ is given by (see Appendix):

$$
\phi_{I}(t)=(\gamma V)^{2} \phi_{Y}(t)=(\gamma V)^{2}\left\{\sum_{i=0}^{1} \sum_{k=0}^{1} y_{i} y_{k} P_{i} \Pi_{i, k}(t)-\left(\sum_{i=0}^{1} P_{i} y_{i}\right)^{2}\right\}
$$


where the stochastic variable $Y$ is defined as being equal to $\kappa$ in state (0) and equal to unity in state (I) $\left(y_{0}=\kappa ; y_{1}=1\right)$. Substitution of equation (2) in (7) yields, after rearrangement

where

$$
\phi_{\bar{Y}}(t)=\bar{Y}^{2} \rho \mathrm{e}^{-t / \tau} ; \quad \phi_{1}(t)=\bar{I}^{2} \rho \mathrm{e}^{-t / \tau},
$$

$$
\begin{aligned}
& \bar{Y}=P_{1}+\kappa P_{0} ; \quad \bar{I}=\gamma V \bar{Y}, \\
& \rho=(\mathrm{I}-\bar{Y})(\bar{Y}-\kappa) / \bar{Y}^{2},
\end{aligned}
$$

$\bar{I}$ being the mean current which flows through the channel, $\bar{Y}$ being the apparent probability of state (I) if one assumes $\kappa=0$ (only one state of non-zero conductance), and $\rho$ being the variance of $Y$ relative to the square of its mean. A regular markovian process, such as the one we have assumed so far, is ergodic. We shall then have, according to the Birkhoff theorem,

$$
\left.\lim _{T \rightarrow \infty} \frac{\mathrm{I}}{T} \int_{0}^{T}\left\{I^{*}\left(t^{\prime}\right)-I^{*}\{\} \bar{I}^{*}\left(t^{\prime}+t\right)-\bar{I}^{*}\right\} \mathrm{d} t^{\prime}=\phi_{I}(t)=\bar{I}^{2} \rho \mathrm{e}^{-t / \tau}, \text { (II }\right)
$$

where the time average on the left-hand side is the autocorrelation function of any actually observed current signal, $I^{*}(t)$, whose average value, $\bar{I}^{*}$, must also coincide with $\bar{I}$. Furthermore, the power spectrum, $S_{I}(f)$, of the signal $I^{*}(t)$, should be given, according to the WienerKhinchin theorem, by

$$
S_{I}(f)=4 \int_{0}^{\infty} \phi_{I}(t) \cos 2 \pi f t \mathrm{~d} t=4 \bar{I}^{2} \rho \tau /\left[\mathrm{I}+(2 \pi f \tau)^{2}\right],
$$

which is known as a Lorentzian spectrum. It is useful for later discussion also to derive from equations (2) the time evolution (relaxation) of the current expectation value, $\langle I(t)\rangle$ starting from state probabilities, $P_{0}^{(0)}$ and $P_{1}^{(0)}$, which are different from the stationary values, $P_{0}$ and $P_{1}$ :

$$
\langle I(t)\rangle=\gamma V\langle Y(t)\rangle=\gamma V \sum_{i=0}^{1} \sum_{k=0}^{1} y_{k} P_{i}^{(0)} \Pi_{i, k}(t) .
$$

From the simple expressions for $\Pi_{i, k}(t)$ in (2), it is easy to verify that:

where

$$
\begin{gathered}
\langle Y(t)\rangle=\bar{Y}+(\langle Y(o)\rangle-\bar{Y}) \mathrm{e}^{-t / \tau}, \\
\langle I(t)\rangle=\bar{I}+(\langle I(0)\rangle-\bar{I}) \mathrm{e}^{-t / \tau}
\end{gathered}
$$

$$
\langle I(0)\rangle=\gamma V\left[\kappa P_{0}^{(0)}+P_{1}^{(0)}\right]=\gamma V\langle Y(0)\rangle \text {. }
$$


Comparison of equation ( 15 ) with (8) illustrates, for the present particular case, that the average relaxation toward equilibrium and the autocorrelation of the spontaneous fuctuations around equilibrium have the same time dependence.

We consider now the case of a membrane containing $M$ independent but identical channels. At any instant of time the total current through the membrane will be the sum of the currents through each separate channel. It is a very simple result of elementary probability theory that the average value of the total current is just $M$ times the average single channel current, given by equation (9). However, the most important result for fluctuation analysis is that also the autocorrelation function and the power spectrum of the total current are simply $M$ times those of each individual channel, given by equations (8) and (12). It is then straightforward to write, for a membrane with $M$ independent two-state channels,

$$
\begin{gathered}
\bar{I}=M \nu V \bar{Y}, \\
\phi_{I}(t)=M^{-1} \bar{I}^{2} \rho \mathrm{e}^{-t / \tau}, \\
S_{I}(f)=M^{-1} \bar{I}^{2} \rho \tau /\left[\mathrm{I}+(2 \pi f \tau)^{2}\right],
\end{gathered}
$$

while the average current relaxation, $\langle I(t)\rangle$, is still described by the same equation (I 5). It is important to stress that $\bar{Y}, \rho$, and $\tau$ in equations (I7)(19), are the same single channel parameters as are defined by (3), (9) and (10). Thus, equations (8) and (9) show that fluctuation analysis yields direct information about single channel parameters even when the number of channels is so large that elementary contributions to the total fluctuations are impossible to resolve. Equation (5) shows, on the other hand, that relaxation analysis yields comparatively poorer information, which concerns only the kinetics $(\tau)$ of the elementary processes and not their size or their number.

Figure I illustrates how power spectrum analysis still leads to simple results despite the increasingly 'noisy' random signals which originate from an increased number of random elementary events. The insets show current fluctuations from a simulated membrane system containing (from bottom to top) $1,4,8$ or 16 two-state channels with $\alpha \simeq \beta$. In the latter case (top trace) single channel fluctuations are already practically inappreciable, and it is easy to realize that a further increase in the number of elementary events would render hopeless any direct analysis of the signals. The main part of Fig. I shows the power spectra 


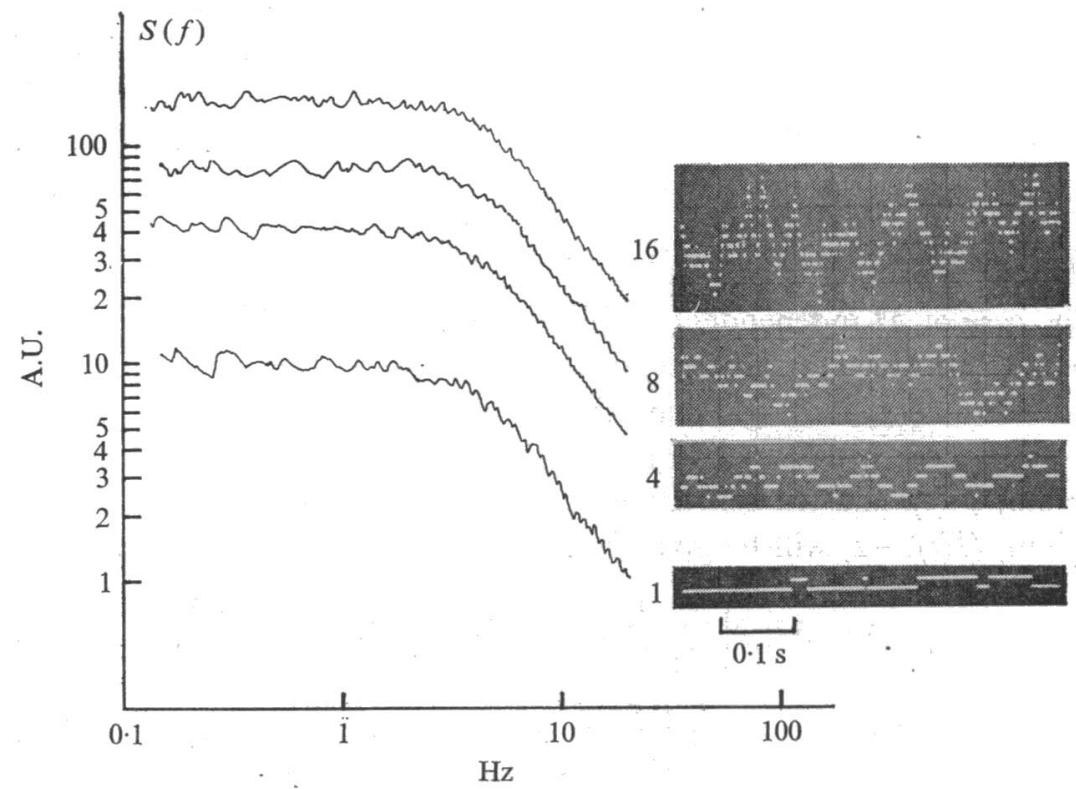

Fig. 1. Random current signals and related power spectral densities in a simulated membrane system containing an increasing number (indicated in the figure) of independent channels undergoing stochastic transitions between open and closed states. The single channel rate constants, $\alpha$ and $\beta$ have approximately the same value $\left(\sim 20 \mathrm{~s}^{-1}\right)$. The amplitudes are in arbitrary units. Notice that the power spectra are merely proportional to each other according to the number of channels present.

obtained from long samples of the signals shown in the insets. All the spectra have exactly the same shape, corresponding to equation 19, with a low frequency amplitude which is merely proportional to the number of channels.

So far we have been considering current fluctuations for a constant voltage across the membrane. The properties of the fluctuations in the voltage across the membrane for a constant current are easily derived from the previous ones. Their power spectrum, $S_{V}(f)$, must be related to $S_{I}(f)$ through the complex membrane impedance, $Z(f)$ (see e.g. Wanke, De Felice \& Conti, 1974):

$$
S_{V}(f)=|Z(f)|^{2} S_{l}(f),
$$

$S_{V}(f)$ is in general no longer a simple Lorentzian since the membrane impedance is rarely frequency independent, particularly in biological membranes or lipid bilayers which have a very large capacitance. 
However, the knowledge of $|Z(f)|^{2}$ allows us in principle to unfold in a simple way the behaviour of $S_{I}(f)$ from that of $S_{V}(f)$. This is one of the contexts in which the spectral analysis offers great advantages with respect to the autocorrelation analysis. No simple relation as equation (20) exists between the voltage and current autocorrelation functions.

\section{Fixed number of multistate channels}

The case of $M$ independent and identical channels, which can exist in more than two states, is conceptually identical to the previous one. If we indicate with $\gamma_{i}$ the single channel conductance in state (i) $(i=1,2, \ldots, r)$ the autocorrelation function of the current fluctuations around equilibrium, $\phi_{I}(t)$, and the relaxation of the average current towards equilibrium, $\langle I(t)\rangle-\bar{I}$, will be given by:

$$
\begin{gathered}
\phi_{I}(t)=M V^{2}\left\{\sum_{i=1}^{r} \sum_{k=1}^{r} \gamma_{i} \gamma_{k} P_{i} \Pi_{i, k}(t)-\left(\sum_{i=1}^{r} P_{i} \gamma_{i}\right)^{2}\right\}, \\
\langle I(t)\rangle-\bar{I}=M V\left\{\sum_{i=1}^{r} \sum_{k=1}^{r} \gamma_{k} P_{i}^{(0)} \Pi_{i, k}(t)-\sum_{i=1}^{r} P_{i} \gamma_{i}\right\},
\end{gathered}
$$

where $P_{i}$ is the stationary probability of state $(i), P_{i}^{(0)}$ is the initial probability of state (i) in the relaxation process, and the transition probabilities $\Pi_{i, k}(t)$ are the solutions of the system of linear differential equations:

$$
\frac{\mathrm{d} \Pi_{i, k}}{\mathrm{~d} t}=\sum_{l=1}^{r} \Pi_{i, l} a_{l k}
$$

with the initial conditions $\Pi_{i, k}=\delta_{i, k}$. The coefficients $a_{l k}$ in equation (23) can be expressed in terms of the rate constants $\alpha_{m n}$, where $\alpha_{m n} \Delta t$ represents the transition probability in time $\Delta t$ from state $(m)$ to state $(n)$ :

$$
a_{k k}=\sum_{m \neq k} \alpha_{k m} ; \quad a_{l k}=\alpha_{k l} \quad[k \neq l] .
$$

Chen \& Hill (1973) have derived formal expressions of $\phi_{I}(t)$ and of its Fourier transform, $S_{I}(f)$, for the most general multistate channel system described by equation (23). The formulas are, however, rather involved and we shall mention here only the most important qualitative results. Quite generally, the solution of (23) leads to an expression for $\left(\Pi_{i, k}(t)-P_{k}\right)$, which is a linear combination with constant coefficients, of $(r-I)$ exponentially decaying functions with time constants solely determined by the rate constants $\alpha_{m n}$, and independent of $i$ and $k$. 
Similar expressions consequently describe the time dependences of $\phi_{I}(t)$ and $\langle I(t)\rangle-\bar{I}$, both being linear superpositions of the $\Pi_{i, k}(t)$ which decay to zero at infinite time. This is again a particular direct demonstration of the equivalence between the time course of fluctuations and relaxations. Finally, the current noise power spectrum, $S_{I}(f)$ is expressed by a linear combination of $(r-r)$ simple Lorentzian spectra.

The multistate channel model which seems most relevant to the discussion of channel noise in nerve membranes can be treated easily without going through the formal solution of equation (23). Following Hill \& Chen (1972a), we consider a generalization of the model which is implicitly assumed in the Hodgkin-Huxley $(\mathrm{HH})$ description of voltageclamp data (Hodgkin \& Huxley, 1952). Each channel contains $x$ independent two-state subunits, each affecting the channel conductance through a multiplicative factor which depends on its state. For this case, the current through a single channel can be written as:

$$
I=\gamma V \prod_{i=1}^{x} Y_{i}
$$

where $\gamma$ is the maximum channel conductance and the stochastic variable $Y_{i}$ takes the value of $\kappa_{i}(<\mathrm{I})$ or unity, depending on whether the subunit $i$ is, respectively, in state (o) (yielding a lower conductance) or in state ( 1$)$. The covariance and the average relaxation of the current, for a membrane containing $M$ (independent) channels of this type, are then derived directly from simple properties of the product of independent variables:

$$
\begin{gathered}
\phi_{I}(t)=(\gamma V)^{2} M\left\{\prod_{i=1}^{x}\left[\phi_{Y_{i}}(t)+\bar{Y}_{i}^{2}\right]-\prod_{i=1}^{x} \bar{Y}_{i}^{2}\right\} \\
=M^{-1} \bar{I}^{2}\left\{\prod_{i=1}^{x}\left[\mathrm{I}+\rho_{i} \mathrm{e}^{-t / \tau_{i}}\right]-\mathrm{I}\right\}, \\
\langle I(t)\rangle=\bar{I}\left\{\prod_{i=1}^{x}\left[\mathrm{I}+\frac{\left\langle Y_{i}(o)\right\rangle-\bar{Y}_{i}}{\bar{Y}^{(i)}} \mathrm{e}^{-t / \tau_{i}}\right]\right\}, \\
\bar{I}=M \gamma V \prod_{i=1}^{x} \bar{Y}_{i} .
\end{gathered}
$$

The autocorrelation $\phi_{I}(t)$, and the average relaxation, $\langle I(t)\rangle$, in equations (26) and (27) will in general contain $2^{x}-1$ different exponentials, and the power spectrum will be a linear combination of $2^{x}-1$ Lorentzians. This is in agreement with the fact that, in general, the number of distinct states of our channel with $x$ subunits is $2^{x}$. 
Three special cases of $(26)$ are particularly relevant to the discussion of current noise in squid giant axons.

For four identical subunits, all characterized by the same rate constants, $\alpha_{n}$ and $\beta_{n}$, and for $\kappa_{1}=\kappa_{2} \ldots=0$, equations (26) and (27) become:

$$
\begin{gathered}
\phi_{I}(t)=M^{-1} \bar{I} 2\left\{\left[\mathrm{I}+\rho_{n} \mathrm{e}^{-t / \tau_{n}}\right]^{4}-\mathrm{I}\right\}, \\
\langle I(t)\rangle=\bar{I}\left[\mathrm{I}+\frac{n(o)-\bar{n}}{\bar{n}} \mathrm{e}^{-t / \tau_{n}}\right]^{4} ; \quad \bar{I}=M \gamma V \bar{n}^{4},
\end{gathered}
$$

where, according to equations (3), (9) and (10), in this particular case, $\rho_{n}=\beta_{n} / \alpha_{n}, n=\langle Y\rangle=\alpha_{n} /\left(\alpha_{n}+\beta_{n}\right), \tau_{n}=\mathrm{I} /\left(\alpha_{n}+\beta_{n}\right)$. The power spectrum corresponding to $(29)$ is given by:

$$
\left.S_{I}(f)=\frac{4}{M} \bar{I}^{2} \tau_{n} \sum_{i=1}^{4}\left(\begin{array}{l}
4 \\
i
\end{array}\right) \rho_{n}^{i} / i\left[1+2 \pi \tau_{n} f / i\right)^{2}\right] .
$$

Equations (29) and (3r) describe the current fluctuations due to the open-close kinetics of the potassium channels in nerve membranes, according to the simplest translation in stochastic terms of the $\mathrm{HH}$ equations, which are based on the description of relaxation (voltageclamp) measurements according to equation (30).

A similar special case of equations (26) and (27) leads to the characterization of the noise from sodium channels, which according to the $\mathrm{HH}$ formulation are assumed to contain three identical subunits with rate constants $\alpha_{m}$ and $\beta_{m}$ and a fourth subunit with rate constants $\alpha_{h}$ and $\beta_{h}, \kappa_{i}$ being zero for all subunits. For this case:

$$
\begin{aligned}
& \phi_{I}(t)=M^{-1} \bar{I}^{2}\left\{\left[\mathrm{I}+\rho_{m} \mathrm{e}^{-t / \tau_{m}}\right]^{3}\left[\mathrm{I}+\rho_{h} \mathrm{e}^{-t / \tau_{h}}\right]-\mathrm{I}\right\}, \\
& \langle I(t)\rangle=\bar{I}\left[\mathrm{I}+\frac{m(\mathrm{o})-\bar{m}}{\bar{m}} \mathrm{e}^{-t / \tau_{m}}\right]^{3}\left[\mathrm{I}+\frac{h(\mathrm{o})-\bar{h}}{\bar{h}} \mathrm{e}^{-t / \tau_{h}}\right] ; \quad \bar{I}=M \gamma V \bar{m}^{3} \bar{h},
\end{aligned}
$$

where $\rho_{m}=\beta_{m} / \alpha_{m}, \rho_{h}=\beta_{h} / \alpha_{h}$, and $m, h, \tau_{m}$ and $\tau_{h}$ correspond to parameters used in the $H H$ equations. The power spectrum corresponding to the autocorrelation function given by (32) can be approximated for $\tau_{h} \gg \tau_{m}$ (as in the case of the $\mathrm{HH}$ equations), as:

$$
\begin{aligned}
S_{I}(f)=\frac{4 \bar{I}^{2}}{\overline{M h}}\left\{(\mathrm{I}-h) \tau_{h}[\mathrm{I}\right. & \left.+\left(2 \pi \tau_{h} f\right)^{2}\right] \\
& \left.+\tau_{m} \sum_{i=1}^{3}\left(\begin{array}{c}
3 \\
i
\end{array}\right) \rho_{m}^{i} / i\left[\mathrm{I}+\left(2 \pi \tau_{m} f / i\right)^{2}\right]\right\} .
\end{aligned}
$$


Finally, for $x$ identical subunits with $\kappa_{1}=\kappa_{2} \ldots=\kappa_{x}=\kappa$, equations (35) and (36) characterize the ' $K$ case' of the generalized model of the potassium channels discussed by Hill \& Chen (1972a) and Chen \& Hill (1973):

$$
\begin{gathered}
\phi_{I}(t)=M^{-1} \bar{I}^{2}\left\{\left[\mathrm{I}+\rho_{n}^{(x)} \mathrm{e}^{\left.\left.-t / \tau_{n}^{(x)}\right]^{x}-\mathrm{I}\right\}}\right.\right. \\
\langle I(t)\rangle=\bar{I}\left[\mathrm{I}+\frac{\left\langle Y_{n}^{(x)}(o)\right\rangle-\bar{Y}_{n}^{(x)}}{\bar{Y}_{n}^{(x)}} \mathrm{e}^{-t / \tau_{n}^{(x)}}\right]^{x}, \bar{I}=M \gamma V\left[\bar{Y}_{n}^{(x)}\right]^{x} .
\end{gathered}
$$

\section{Fluctuations in channel number}

The most general case of channel noise from independent channels also involves fluctuations in the number of existing channels. This further complication may for example be required in artificial lipid bilayers which may exchange channel-inducing substances with the bathing aqueous solutions. In addition to the rate constants for internal conversion it is then necessary to specify the channel formation rates in state (i), $\lambda_{i}$, and the disappearance rate constants, $\beta_{i 0}, \beta_{i 0} \Delta t$ being the probability that a channel in state (i) disappears in time $\Delta t$. The autocorrelation function will again be of the form of a linear combination of exponentials. However, for $r$ internal channel states we shall have in this case $r$ (not $r-I$ ) time constants: all of which will depend both on the internal conversion rate constants, $\alpha_{i j}(i, j=1,2, \ldots, r)$ and on the disappearance rate constants $\beta_{i 0}(i=\mathrm{I}, 2, \ldots, r)$. The formation rates, $\lambda_{i}$, will influence the average number of channels in the various states, and, therefore, the coefficients of the various exponentials in the autocorrelation function. A simple special case is obtained when, for any state (i),

$$
\beta_{i 0} \ll \sum_{k \neq i, 0} \alpha_{i k} .
$$

This condition implies that the lifetime of a channel in any state is long enough to allow equilibration among all possible internal channel states. It can be shown that the current autocorrelation function for this special case is:

$$
\phi_{I}(t)=\bar{M}\left\{\phi_{I_{1}}(t)+\bar{I}_{1}^{2} \exp -t \sum_{i=1}^{r} P_{i} \beta_{i 0}\right\}
$$

where $\bar{M}$ is the total average number of channels present at any time, independently of their internal state; $\phi_{I_{1}}(t)$ and $\bar{I}_{1}$ are the covariance and the average value of the current which would flow through a single, permanent, channel; $P_{i}$ is the equilibrium probability of state (i) for 
such a permanent channel. The first contribution to $\phi_{I}(t)$ in equation (37) is identical to what is expected from $\bar{M}$ permanent channels. The second contribution is due to the formation and disappearance of channels carrying an average current $\bar{I}_{1}$. For channels with only one state:

$$
\phi_{I}(t)=\bar{M} I_{1}^{2} \mathrm{e}^{-\beta t}=\bar{M}^{-1} \bar{I}^{2} \mathrm{e}^{-\beta t},
$$

where $I_{1}$ is the current through a single channel and $\beta^{-1}$ is the average channel lifetime.

\section{Channel noise modulating intrachannel noise}

So far we have considered only the noise associated with fluctuations in the conductance or in the number of channels, and we have assumed that the current flowing through a channel in any fixed configuration is constant, free of what we may call intrachannel noise. Such assumption cannot be maintained in general, since thermal, shot and $\mathrm{I} / f$ noise are expected to be present anyway, even if the channels are stable single state pores. However, as shown below, our preceding simplified analysis is justified by the fact that, under certain conditions, channel noise and intrachannel noise are expected to be additive to one another.

Let us first consider the case of channels, with only one non-zero conductance value and assume that the current, $I$, through a single channel can be written as the product of two independent stochastic variables:

$$
I=\mathcal{J} \cdot Y,
$$

where the fluctuations of $J$ account for intrachannel noise and $Y$ fluctuates between zero and unity. The assumption that the stochastic fluctuations of $J$ are not influenced by the random switching of channel state is very reasonable for thermal or shot noise, which have very short correlation times. However, for $\mathrm{I} / f$ noise the independence of $J$ and $Y$ has no obvious justification other than that it predicts a I $/ f$ noise power proportional to the square of the average current, as observed experimentally in nerve membranes. From a simple property of the covariance of the product of independent variables, it follows from equation (39):

$$
\phi_{I}=\left(\phi_{J}+\bar{J}^{2}\right)\left(\phi_{Y}+\bar{Y}^{2}\right)-(\bar{J} \bar{Y})^{2}=\bar{J}^{2} \phi_{Y}+\bar{Y}^{2} \phi_{J}+\phi_{J} \phi_{Y},
$$

For thermal noise or shot noise we expect $\phi_{I}$ to decay to zero much faster than $\phi_{Y}$ so that $\phi_{J} \phi_{Y} \simeq \phi_{J} \phi_{Y}(0)$, and:

$$
\phi_{I} \cong \bar{J}^{2} \phi_{Y}+\phi_{J}\left[\phi_{Y}(0)+\bar{Y}^{2}\right]=\bar{J}^{2} \phi_{Y}+\left\langle Y^{2}\right\rangle \phi_{J}=\bar{J}^{2} \phi_{Y}+\bar{Y} \phi_{J}
$$


where the last equality derives from the fact that, in the present simple case, $(Y \equiv\{0,1\}),\left\langle Y^{2}\right\rangle=\bar{Y}$.

For $\mathrm{I} / f$ noise, we expect $\phi_{Y}$ to decay much faster than $\phi_{J}$ so that $\phi_{J} \phi_{Y} \simeq \phi_{J}(0) \phi_{Y}$, and

$$
\phi_{I}=\left\langle i^{2}\right\rangle \phi_{X}+\bar{X}^{2} \phi_{i}
$$

It can be shown, with a slightly more involved argument, that equation (4I) is more generally applicable to the case of an arbitrary number of channel conductance states, under the only assumption that the correlation times of $Y$ are much longer than those of thermal or shot noise. Rather than the independence of $J$ and $Y$ one should require in this case that the thermal or shot noise, during each channel state lifetime, have powers proportional respectively to the channel conductance or to the average current.

\section{EXPERIMENTAL TECHNIQUES}

The major technical problem in noise measurements is that of minimizing all possible sources of fluctuations that are not intrinsic to the system under study. Typical sources of unwanted disturbances are microphonics, electromagnetic pick-up, fluctuations in junctional emfs, (either metal-metal or metal-electrolyte), and amplifier noise. All these sources can be reduced through a careful design of the experimental apparatus. Alternatively, or in addition, ways of subtracting extraneous noise from the noise under study can often be found.

In membrane studies, electrode noise is of particular concern, since it is not easily removed a posteriori. $\mathrm{Pt}-\mathrm{Pt}$ black electrodes with a low impedance down to low frequencies are particularly recommended, as an alternative to $\mathrm{Ag}-\mathrm{AgCl}$ (Wanke et al. 1974).

Amplifier noise deserves special discussion. Design techniques exist for assemblage of available components for lowest noise performance (see e.g. Motchenbauer \& Fitchen, 1973), but the lowest attainable levels of amplifier noise depend ultimately on the quality of the commercially available solid-state devices. The input stage is usually the most critical part of the set-up, and selected junction field effect transistors (JFET) should be employed for it. Apart from the above general comments, the problem of amplifier noise presents different aspects for different membrane preparations, and depending on whether current noise or voltage noise is measured. These have been discussed 


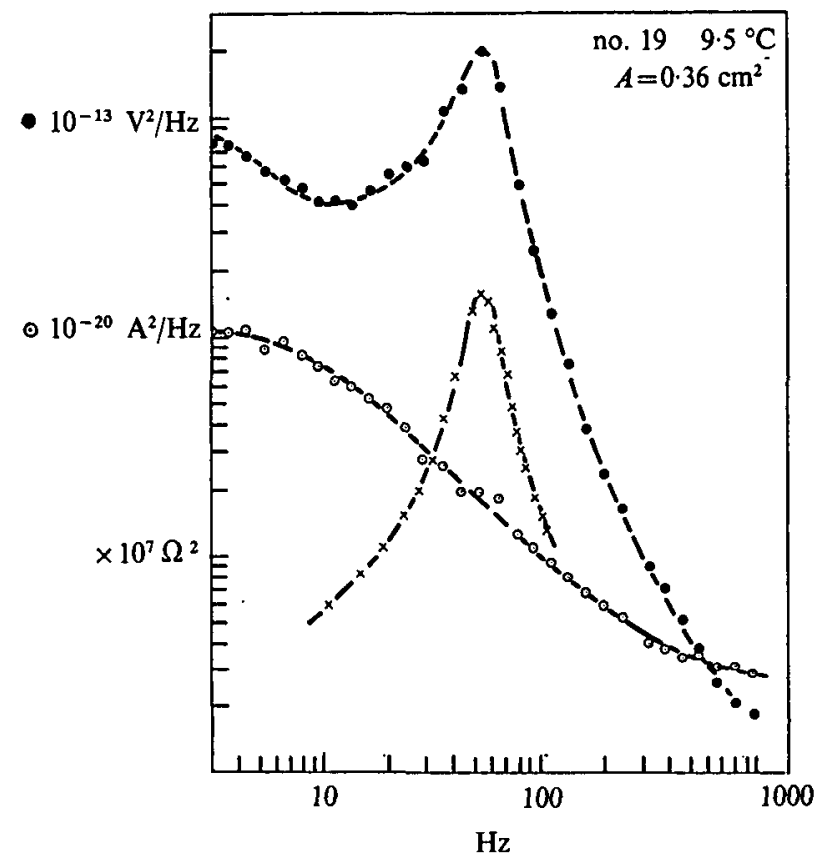

Fig. 2. Current noise power spectrum, $O$, voltage noise power spectrum, -, and the square modulus of the impedance, $x$, in a large area $\left(0.36 \mathrm{~cm}^{2}\right)$ of squid axon membrane at resting potential in normal physiological conditions. Temperature: $9{ }^{\circ} \mathrm{C}$. (From Wanke et al. 1974.)

in detail by various authors, for example Derksen (1965) and Siebenga, Meyer \& Verveen (1973) for frog node preparations; Poussart (197I) and Fishman (1973, 1975) for artificial node preparations in giant axons; Wanke et al. (1974), De Felice, Wanke \& Conti (1975), and Conti, De Felice \& Wanke (1975) for large areas of squid axon membrane.

The difference between voltage-noise and current-noise measurements is worth stressing here. As already mentioned, the two measurements yield in principle the same information provided the membrane impedance locus is known (equation (20)), but $S_{I}(f)$ can be directly related to channel noise, without requiring supplementary impedance studies. $S_{I}(f)$ and $S_{V}(f)$ are expected to be quite different particularly in well isolated areas of nerve membrane, whose impedance varies strongly in the range of frequencies where channel noise is studied (Wanke et al. 1974). This is illustrated in Fig. 2, showing plots of experimental measurements of $S_{V}(f), S_{I}(f)$ and $|Z(f)|^{2}$, from the same preparation of squid 
giant axon. The contrasting result reported by Fishman (1973), that current noise and voltage noise spectra from very small patches of squid axon membrane are practically identical, must be due to the large shunt conductance in parallel with the artificial node (Fishman, I975), creating a poor isolation of the node (see also Pooler \& Oxford, 1972). Invasion of currents from lateral regions bordering the gap might seriously impair in this preparation the possibility of actually clamping either the current or the voltage in the central area under study.

The advantage of obtaining more direct information from current noise is obtained at the expense of simplicity of the measuring setup. The measurements must be performed under voltage-clamp conditions using special feedback circuits whose specifications have been discussed in detail by Poussart (1971, 1973).

When some freedom in selecting the membrane area under study is allowed, the problem of optimizing such area for best membrane noise detection is of some importance. It can be shown quite generally (see e.g. De Felice et al. 1975) that the maximum ratio between membrane noise and amplifier noise is obtained when: $|Z|^{2}=\mathrm{e}_{n}^{2} / i_{n}^{2}$. Here $Z$ is the membrane impedance, and $\mathrm{e}_{n}^{2}$ and $i_{n}^{2}$ are the voltage and current spectral densities of the amplifier input noise. The above relationship cannot in general be satisfied at all frequencies, but it leads anyhow to an estimate of the optimal range of membrane impedances and, therefore, of membrane areas. It should be stressed, however, that such optimization is not always critical, while other considerations may actually make it preferable to depart considerably from it. For example, typical figures for the input noise of commercially available electronic devices

$$
\left(\mathrm{e}_{n}^{2} \sim 1 \mathrm{IO}^{-16} V^{2} \mathrm{~s} ; i_{n}^{2} \sim 1 \mathrm{IO}^{-28} \mathrm{~A}^{2} \mathrm{~s}\right)
$$

lead to optimal membrane impendances of the order of $\mathrm{I} M \Omega$. For the squid axon membrane $\left(|Z| \sim \mathrm{I} K \Omega \mathrm{cm}^{2}\right.$ at low frequencies, and $|Z| \sim 160 \Omega \mathrm{cm}^{2}$ at $\mathrm{I} \mathrm{KHz}$ ), this corresponds to an optimal area of $10^{-3} \mathrm{~cm}^{2}$ or less, and such an estimate seems to have been the leading motivation for the use of artificial nodes (Fishman, 1975). However, recent experiments (Wanke et al. 1974; De Felice et al. 1975; Conti et al. 1975) have shown that the requirement of optimal area can be freely disobeyed without seriously impairing good membrane noise detection. Working with much larger membrane areas $\left(\sim 0.3 \mathrm{~cm}^{2}\right)$ leads to great advantages, such as an easier nerve preparation to set up, with better control of its physiological and isolation properties. 
The analysis of the random noise signals may constitute a major part of noise experiments. However, a discussion of the problems involved in signal processing (e.g. anti-aliasing filtering, sampling, time windows, etc.) is beyond the scope of the present review and can be found elsewhere (e.g. Rabiner \& Rader, 1972). Furthermore, present day noise analysis is often greatly simplified by the availability of special purpose machines, such as correlators or power spectrum analysers, which can process directly on-line the analogue random signal, having all the necessary processing steps built in. Alternatively, analogue data stored on magnetic tape can easily be processed with digital computers using standard available software (e.g. the Fourier Analyzer System HP 545IA). A general theoretical result which is worth keeping in mind is that the accuracy of noise data increases as the square root of the duration, $T$, of the analysed noise sample. Thus, the ratio between the standard deviation and the average value of the measured noise power in the frequency interval, $\Delta f$, is approximately equal to $(T \Delta f)^{-1 / 2}$ (Rice, 1944).

Whether to analyse noise signals in terms of autocorrelation functions or power spectra may be in many cases a matter of convenience or taste. However, in studies of nerve membranes, power spectra are largely preferred, since they allow an easy separation of channel noise from I $/ f$ noise, and even when correlation analysis is used, the data are finally converted into power spectra through a Fourier transformation (Siebenga et al. 1973; Siebenga, De Goede \& Verveen, 1974). On the other hand, the noise from lipid bilayers containing gramicidin A does not show a significant $I / f$ component, and has preferably been analysed in terms of its autocorrelation function (Neher \& Zingsheim, 1974; Zingsheim \& Neher, I974; Kolb et al. 1975).

\section{NOISE FROM NERVE MEMBRANES}

\section{Early studies (I/f noise and burst noise)}

The first studies of membrane noise, performed on the node of Ranvier of isolated frog sciatic nerve fibres, were published in 1965 (Derksen, I 965 ; Verveen \& Derksen, I965). In addition to thermal noise, the membrane voltage was found to contain a large excess noise of the $\mathrm{I} / f$ type in the range of frequencies from 0.1 to $10000 \mathrm{~Hz}$. Compelling evidence suggested that the $\mathrm{I} / f$ noise was mainly associated with the 
passive movement of potassium ions, and this result was confirmed by later experiments on the same preparation (Derksen \& Verveen, 1966; Verveen $\&$ Derksen, 1968; Siebenga \& Verveen, 1970) and also on giant axons of lobsters (Poussart, I97). A detailed discussion of $r / f$ noise in nerve membranes is given by Verveen \& De Felice (1974). We believe that quantitative experimental studies of $\mathrm{I} / \mathrm{f}$ noise, together with a better theoretical understanding of its origin, will eventually yield some information about ion-ion and ion-channel interactions in the ionic channels of nerve membranes, but clear results in this direction are still missing.

The measurements of small voltage fluctuations in frog nodes showed also the presence of another type of excess noise which became generally evident only for large hyperpolarizations, in the form of irregularly occurring miniature depolarizing potentials (Derksen, 1965; Verveen \& Derksen, 1968, 1969). This was termed by the above authors 'burst noise'. It was found to be independent of the extracellular concentration of potassium and of resting potential, while substitution of sucrose for extracellular $\mathrm{NaCl}$ shifted its appearance toward higher hyperpolarizations. From these observations it was speculated that burst noise could be associated to the passive movement of sodium ions, or even with the random opening of sodium channels (Verveen \& Derksen, 1969). The latter interpretation seems untenable since burst noise increases, while the probability of opening of sodium channels decreases, with increased hyperpolarization. The absence of any effect of the sodium channel blocking drug, tetrodotoxin (TTX), upon burst noise (Siebenga et al. 1974), leads to the same conclusion (Verveen $\&$ De Felice, 1974). The association of burst noise with sodium ion flow was suggested mainly by the $\mathrm{NaCl}$ versus sucrose substitution experiment. However, it is known (Conti, Fioravanti \& Wanke, 1973) that lowering the ionic strength of the extracellular solution increases drastically the membrane potential at which (macroscopic) membrane dielectric breakdown occurs. Thus, the results of such experiments are also consistent with the hypothesis that the bursts are transient localized dielectric breakdowns (Del Castillo \& Katz, 1954). Such an hypothesis is further supported by the observation of very similar phenomena in simple lipid bilayers (Yafuso, Kennedy \& Freeman, 1974). 


\section{Evidence for channel noise}

In more recent years, experiments on depolarized nodes of Ranvier have revealed the presence of other additional voltage noise components which could be described fairly well as simple Lorentzian noise added on top of $\mathrm{I} / f$ noise (Siebenga \& Verveen, 1971, 1972; Siebenga et al. 1973, 1974). The order of magnitude, and the temperature dependence of the Lorentzian cut-off frequency were found to be in qualitative agreement with the expected behaviour of the noise due to fluctuations in the conductance state of the ionic channels governing the late (potassium) voltage-clamp current. The most compelling evidence for the identification with potassium channel noise was the sensitivity to the addition of tetraethylammonium ions (TEA) (Siebenga et al. 1974). A quantitative account of the observed Lorentzians in terms of the $\mathrm{HH}$ description of relaxation (voltage-clamp) experiments, was not given. As discussed below, this requires the direct comparison of voltage-clamp and noise data from the same preparation, and according to the same microscopic model of channel kinetics. Thus, the potassium channel density of $1000 \mu \mathrm{m}^{-2}$, estimated by Siebenga et al. (1973) (according to a model implying only two channel states of equal probability) although indicative, might be very inaccurate. Using a more general model, in which each channel is assumed to have only one possible nonzero conductance value (but an arbitrary number of states), Begenisich \& Stevens (1975) have obtained an estimate of the single potassium channel conductance, from their measurements on frog nodes, of $4 \times 10^{-12} \mathrm{~S}$. This value is about six times smaller than the average estimate of Siebenga et al. (1973).

Very recently it has also been reported that the Lorentzian noise component expected to be associated with the $h$ process in sodium channels (the first component in equation (34)) can be observed in depolarized frog nodes after blocking the potassium current with intracellular caesium ions and TEA (Van den Berg, 1975). Although detailed data have not been published yet, the results seem to be in good agreement with the simple $\mathrm{HH}$ model and to lead to a correct estimate $\left(2-4 \times 10^{-12} \mathrm{~S}\right)$ of the single sodium channel conductance (see later).

Observations of channel noise have been reported also for artificial nodes (Fishman, 1973, 1975) and for large voltage-clamped areas (Wanke et al. 1974; De Felice et al. 1975 ; Conti et al. 1975) of the squid 
giant axon. The results of the latter experiments will be discussed in detail in the next section. As mentioned in experimental techniques (pp. 467-8), the sugar gap artificial node preparation is subject to criticism, there being doubts about the unequivocal correlation between the observed 'humps' in the noise spectra, and potassium channel noise (Wanke et al. 1974). It seems that, with such a technique, what one really measures are the voltage fluctuations across the shunt resistance in the gap regions, due to current fluctuations both in the central (clamped) area and in the lateral (uncontrolled) regions. Thus, while Fishman's observations may indicate qualitatively the presence of potassium channel noise in squid axons, it seems clear that the results are very difficult to interpret quantitatively.

\section{Channel noise in voltage-clamped squid giant axons}

Noise measurements from long $(\sim 20 \mathrm{~mm})$ segments of squid giant axons, well isolated from lateral regions by air-gaps, and kept under good space- and voltage-clamp conditions, can be performed using techniques described elsewhere (Wanke et al. 1974; De Felice et al. 1975; Conti et al. 1975). In addition to the good control of its physiological and isolation properties, this preparation offers major advantages such as the possibility of simultaneous noise and average current measurements, together with an independent continuous monitoring of the absolute membrane potential. Furthermore, measurements of large voltage-clamp currents under exactly the same conditions and possibly in the same axon, allow a direct comparison between macroscopic relaxation and noise data, needed for quantitative analysis of the results.

A typical current noise power spectrum, $S_{I}(f)$, from an axon voltage clamped near its resting potential at a temperature of $9{ }^{\circ} \mathrm{C}$ is shown in Fig. 3. The figure contains also a plot of the membrane impedance modulus squared, $\left|Z_{m}\right|^{2}$, measured from small sine wave analysis (Conti, 1970), from which the amplifier's noise contribution, $\mathrm{e}_{n}^{2} /\left|Z_{m}\right|^{2}$, and the theoretical thermal noise, $4 k T \operatorname{Re}\left(Z_{m}\right)^{-1}$, could be evaluated. It is seen that, apart from a small correction for frequencies above $200 \mathrm{~Hz}$ most of the measured noise can be attributed to excess (non-thermal) membrane noise shown in the figure as $S_{I}{ }_{I}^{(c)}(f)$. Fitting the power spectrum of Fig. 3 with a straight line would lead to a slope very close to - I showing the presence of a major I/f noise component. However, significant departures from such a straight line are also apparent and were 


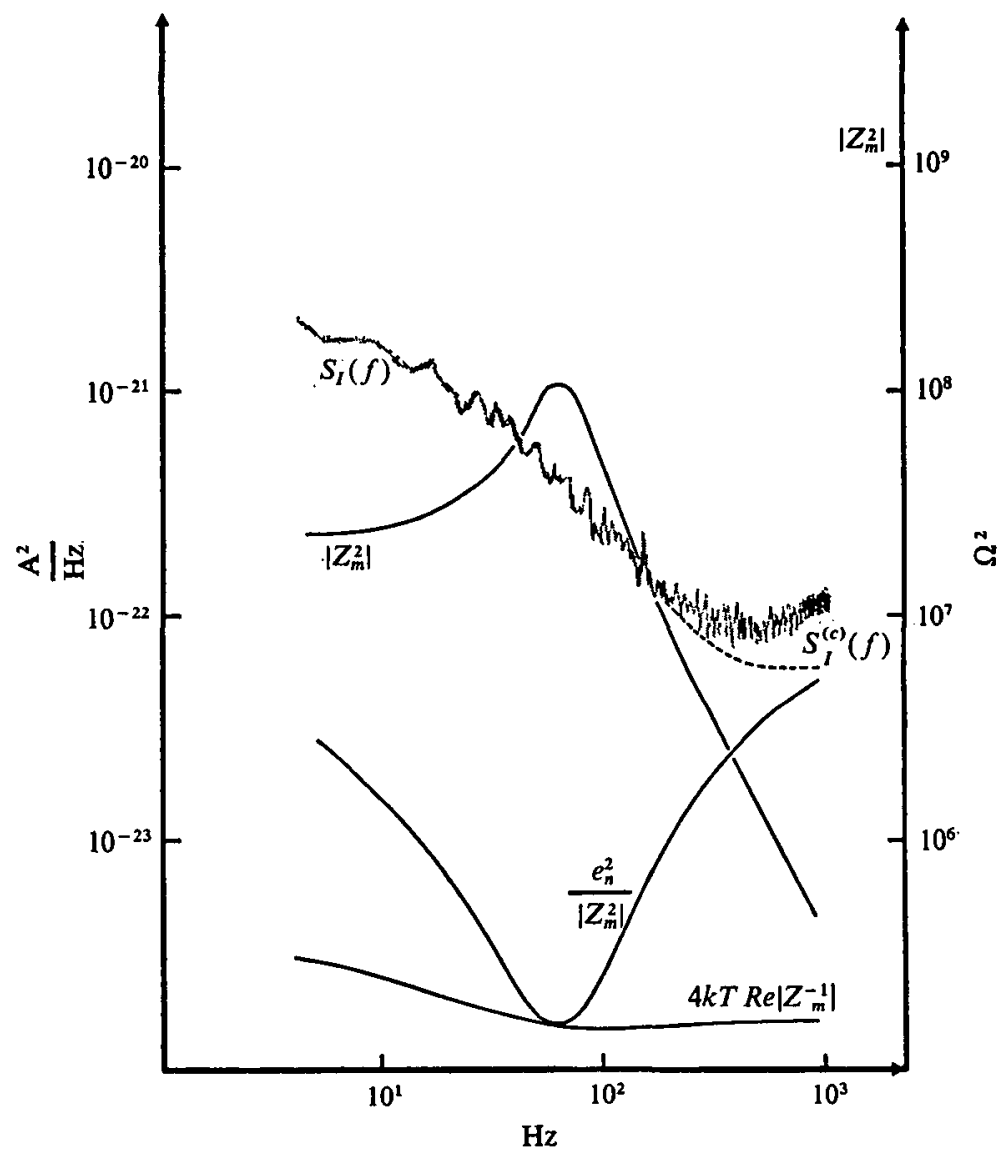

Fig. 3. Current noise spectral density, $S_{I}(f)$, and the square amplitude of the impedance, $\left|Z_{m}\right|^{2}$, in a squid axon membrane, plotted together with the expected Nyquist noise, $4 k T \operatorname{Re} Z_{m}{ }^{-1}$, and amplifier noise, $e_{n}^{2} /\left|Z_{m}\right|^{2}$. The dashed line, $S_{l}^{(c)}(f)$, gives the membrane current noise spectrum corrected for the latter contribution. Temperature: $9{ }^{\circ} \mathrm{C}$. Membrane area $0.32 \mathrm{~cm}^{2}, V_{m}=-56 \mathrm{mV}$. (From Conti et al. 1975.)

attributed to channel noise modulation of intrachannel, mainly $\mathrm{I} / f$, noise (Conti et al. 1975).

According to the $\mathrm{HH}$ analysis of voltage-clamp experiments (Hodgkin \& Huxley, 1952) and following the theory previously discussed, the power spectrum, $S_{I}^{(c)}(f)$ should be dissected into the sum of three components with distinct spectral characteristics. I/ $f$ noise, as a general characteristic of passive ionic fluxes (De Felice \& Firth, 1970; Hooge \& Gaal, 1970; De Felice \& Michalides, 1972; Michalides, Wallaart \& De Felice, 1973) is expected to affect the currents flowing through the 


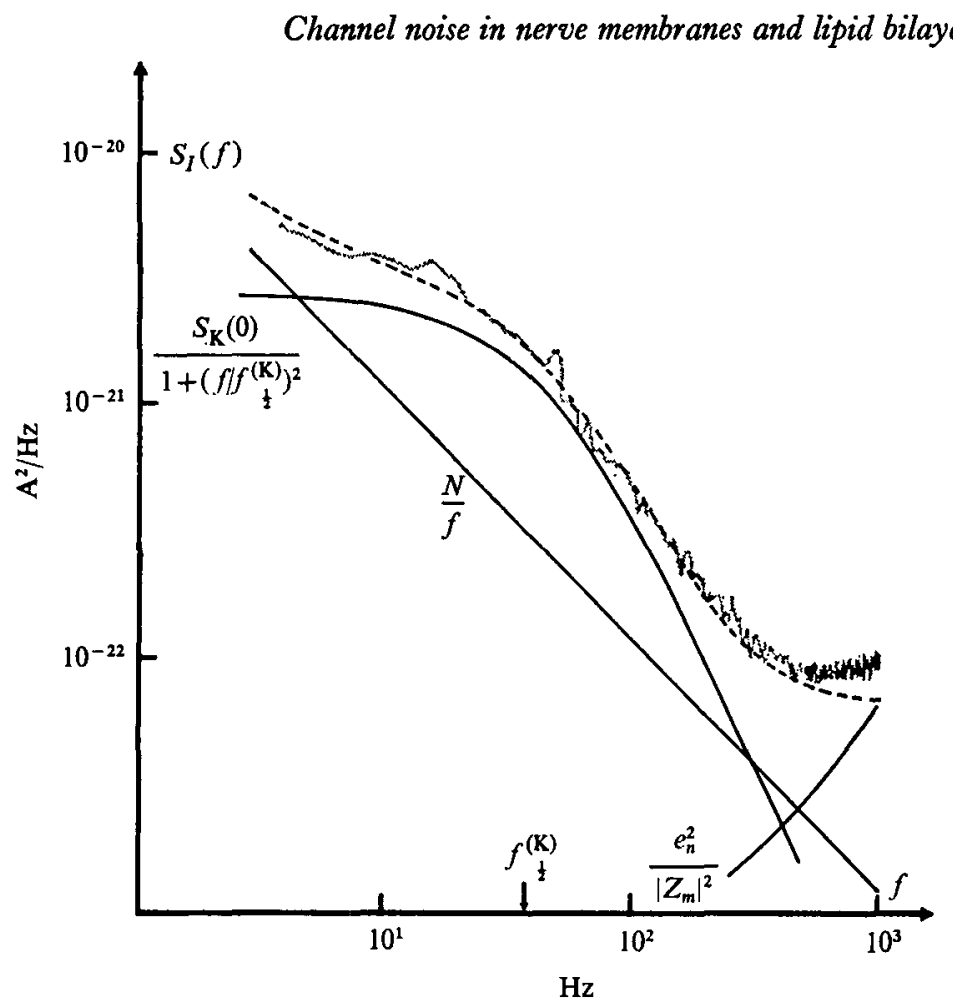

Fig. 4. Current noise spectral density $S_{I}(f)$ from a squid axon treated with $20 \mathrm{nM}$ TTX at $V_{m}=-53 \mathrm{mV}$. The dashed line is the sum of a Lorentzian, a $I \mid f$ component and the amplifier noise $e_{n}^{2} /\left|Z_{m}\right|^{2}$. The three separate components ars drawn as continuous lines. Temperature: $9^{\circ} \mathrm{C}$. Membrane area $0.28 \mathrm{~cm}^{2}$. (From Conti et al. 1975.)

leakage pathway and through the potassium or sodium selective channels. The random opening and closing of these channels is further expected to produce current fluctuations with power spectra which are sums of a small number of Lorentzians (see equations (3I) and (34)). Distinct spectral characteristics of the sodium versus potassium channel noise are expected on the basis of the different relaxation times which characterize the kinetics of the two types of channels (see e.g. Fig. 3.32 of Cole, 1968).

$K$ channel noise. The actual extraction of potassium channel noise, $S_{K}(f)$, from $S_{I}^{(c)}(f)$ can be most easily performed by eliminating sodium currents with TTX (Narahashi, Moore \& Scott, r964) and approximating the sum of Lorentzians in equation (3I) with a simple Lorentzian. In the range of membrane voltages explored by Conti et al. (1975), this approximation seems fairly justified according to the actual values for 


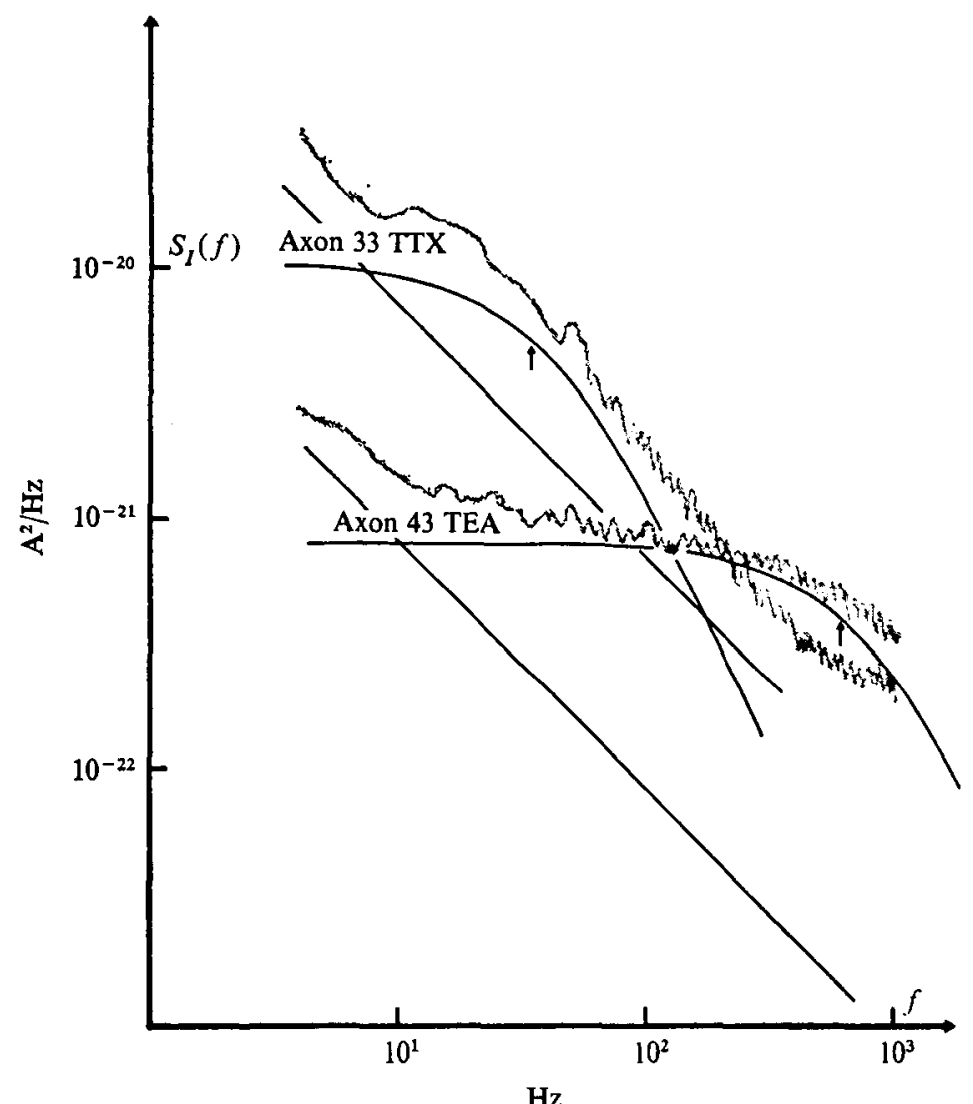

Fig. 5. Current noise spectral density for $V_{m}=-4 \mathrm{ImV}$, from an axon injected intracellularly with $70 \mathrm{mM}$-TEAchloride, compared with the spect rum at the same $V_{m}$ from an axon treated with $20 \mathrm{nM}$ TTX. The two axons had approximately the same area $\left(0.31 \mathrm{~cm}^{2}\right)$ and the two spectra were obtained at the same temperature, $9^{\circ} \mathrm{C}$. The continuous lines show the two components ( $\mathrm{I} / f+$ Lorentzian) whose addition approximates each spectrum. The arrows indicate the half power points of the Lorentzian components. (From Conti et al. 1975.)

the potassium channel parameters extracted from voltage-clamp data. $S_{K}(f)$ can then be extracted from TTX treated axons by expressing the total noise (apart from amplifier noise correction) as the sum of a I/f component and a simple Lorentzian. This procedure is illustrated in Fig. 4. It is seen that the fitting of the measured spectrum with the sum of the three solid lines, representing $\mathrm{I} / f$, Lorentzian and amplifier noise, is quite good down from $5 \mathrm{~Hz}$ up to $500 \mathrm{~Hz}$. The slight discrepancy in the range from $500 \mathrm{~Hz}$ to $\mathrm{I} \mathrm{KHz}$ could be due to a slightly wrong 
estimate of the amplifier noise, which becomes predominant at such frequencies. Should it have any real significance it would constitute anyhow a minor feature of the overall spectrum.

An independent check that $S_{K}(f)$, extracted with the above procedure is associated with $K$ channel noise is obtained by studying the noise from axons containing intracellular concentrations of TEA sufficient to block the potassium currents completely (Tasaki \& Hagiwara, 1957; Armstrong \& Binstock, 1965 ). Fig. 5 shows the current noise power spectrum from an axon treated with $70 \mathrm{mM}$-TEA and held at a membrane potential, $V_{m}$, of $-4 \mathrm{I} \mathrm{mV}$, as compared with the spectrum from a TTX-treated axon at the same $V_{m}$ and temperature $\left(9^{\circ} \mathrm{C}\right)$. The whole low-frequency region of the TTX spectrum is seen to be strongly depleted by TEA. On the other hand, the TEA spectrum becomes larger than that of TTX at high frequencies, a feature which is expected from the presence of the sodium channel noise, discussed below.

At the level of approximation implicit in the extraction procedure of Fig. $4, S_{K}(f)$ is fully characterized by its low frequency limit, $S_{K}(0)$, and by the cut-off frequency, $f_{\frac{K}{2}}^{\left(\frac{K}{2}\right)}$, at which $S_{K}\left(f_{\frac{K}{2}}^{(K)}\right)=S_{K}(0) / 2$. In agreement with the theoretical expectation of the simple model leading to equation (3I), the experimental results of Conti et al. (1975) showed a temperature dependence of $S_{K}(0)$ or $f_{\frac{1}{2}}^{(K)}$, which was very close to that of $\tau_{n}\left(Q_{10} \sim 3\right)$ or $\mathrm{r} / \tau_{n}$. The slight dependence of $f_{\frac{1}{2}}^{(K)}$ on membrane potential and the range of its experimental values also agreed with the simple $\mathrm{HH}$ kinetic model. Furthermore, the strong voltage dependence of $S_{K}(0)$ could be fitted fairly well according to (3I), using the $\mathrm{HH}$ voltage-clamp parameters appropriately extracted from the same giant axon preparation. The fitting obtained by Conti et al. (1975) is shown by the dots and the solid line in Fig. 6, where similar data for the sodium channel noise, to be discussed below, are also reported. Since, for any model of independent channels, the absolute value of the current noise power for a fixed average current is simply inversely proportional to the number of channels, such fitting leads to an estimate of the density of potassium channels, $M_{K}$, which, according to the simplest $\mathrm{HH}$ model, was found to be of the order of $60 \mu \mathrm{m}^{-2}$. This estimate was little affected by the particular model assumed for the potassium inactivation process (Ehrenstein \& Gilbert, 1966 ), which is anyhow small in the range of $V_{m}$ applicable to the data of Fig. 6. A discussion of how critical, for the estimate of $M_{K}$, the assumption of the simple $\mathrm{HH}$ channel kinetics is, and of the relevance of the above results for restricting the 
realm of models with plausible channel kinetics is given later. From the measured maximum potassium conductance, $\bar{g}_{K} \simeq 7 \times 10^{-2} S \mathrm{~cm}^{-2}$, and the estimated channel density, $M_{K}=60 \mu \mathrm{m}^{-2}$, the conductance of one open potassium channel in the axon of Loligo vulgaris is of the order of $12 \times 10^{-12} \mathrm{~S}$.

$N a$ channel noise. The separation of the Na channel noise, $S_{N a}(f)$ can be obtained with two different methods. In axons injected intracellularly with large concentraions of TEA only $\mathrm{I} / f$ noise and sodium channel noise are expected to be present. The total noise from these preparations (after amplifier noise correction) was approximated by Conti et al. (1975) with the sum of a I/f spectrum plus a Lorentzian, this latter component being attributed to Na channel noise. Alternatively one can subtract the noise power spectrum obtained in TTX from that obtained in normal sea water, from the same axon at the same temperature and the same membrane potential. This differential method is largely preferable, since it requires neither accurate estimates of amplifier noise (which is cancelled automatically) nor assumptions about the shape of the expected sodium channel noise. In the experiments reported by Conti et al. (1975), a difference spectrum could be determined with reasonable accuracy for frequencies larger than about $100 \mathrm{~Hz}$ and it showed in that range a pseudo-Lorentzian shape without significant $r / f$ noise contribution.

Both methods yielded similar results in terms of the relatively low ( 100 Hz) frequency limit, $S_{N a}(0)$, of the channel noise spectrum and of its cut-off frequency, $f_{\frac{1}{2}}^{(\mathrm{Na})}$. The data were compared with what was expected from the second component in equation (34), involving essentially only the $m$ process of the $\mathrm{HH}$ model. The first component, related to the $h$ process, could in fact be disregarded above $100 \mathrm{~Hz}$. As in the case of the potassium channel noise, the temperature and voltage dependence of $S_{N a}(0)$ and $f_{\frac{1}{2}}{ }^{(N a)}$, were found to be in good agreement with the theoretical expectations from the simple $\mathrm{HH}$ model. The plot of $S_{N a}(0)$ data is shown in Fig. 6 (triangles) together with the theoretical curve (interrupted line).

From the absolute values of $S_{N a}(\circ)$ the density of sodium channels, $M_{N a}$, was estimated to be about $330 \mu \mathrm{m}^{-2}$. This figure is fairly close to those (ranging from 400 to $500 \mu \mathrm{m}^{-2}$ ) obtained in the last few years by various authors using three different experimental approaches, other than noise. These include measurements of gating currents (Armstrong 


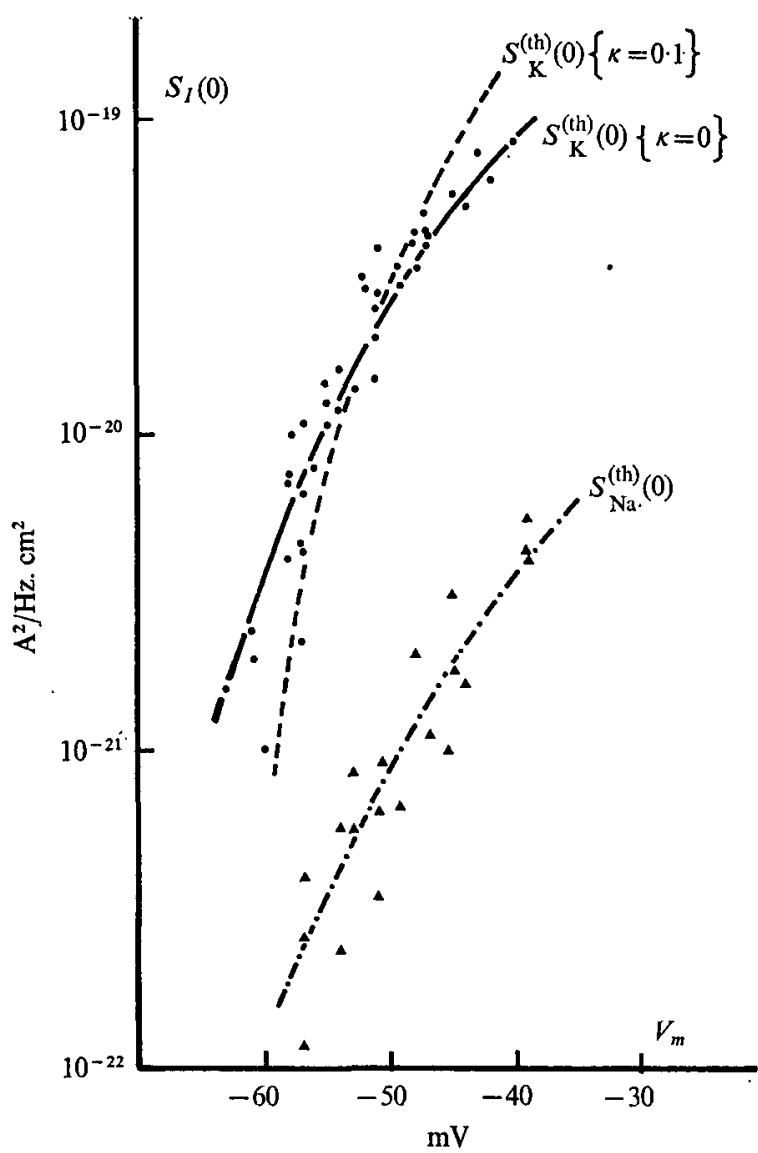

Fig. 6. Plots of the low frequency spectral density of potassium, $\boldsymbol{O}$, and sodium, $\Delta$, channel noise in squid giant axons, against membrane potential. The data, obtained from many axons, are all scaled to $6{ }^{\circ} \mathrm{C}\left(Q_{10}=3\right)$ and to $1 \mathrm{~cm}^{2}$ of membrane area. The continuous and the interrupted theoretical curves give the expectations from the simple $\mathrm{HH}$ channel kinetics assuming $M_{K}=60 \mu \mathrm{m}^{-2}$ and $M_{N a}=330 \mu \mathrm{m}^{-2}$ respectively.

The dashed line shows the theoretical expectation according to a four subunits ' $\kappa$ model' for the potassium channels (Hill \& Chen, 1972) with $\kappa=0.1$ and $M_{R}=40 \mu \mathrm{m}^{-2}$. (Modified from Conti et al. 1975.)

\& Bezanilla, 1974; Rojas \& Keynes, 1975), of TTX uptake and release kinetics (Keynes et al. 1975), and of tritiated TTX binding (Levinson $\&$ Meves, I975). From the measured maximum sodium conductance, $\bar{g}_{N a}=0.13 S \mathrm{~cm}^{-2}$, the conductance, $\gamma_{N a}$, of one open sodium channel in giant axons of Loligo vulgaris was estimated to be about $4 \times 10^{-12} S$, roughly 3.5 smaller than that of potassium channels. It is interesting 
to notice that approximately the same $\gamma_{N a}$ has been obtained in frog nodes, from measurements of gating currents, despite the fact that in this preparation $M_{N a}$ is estimated to be one order of magnitude higher than in squid axons (Nonner, Rojas \& Stämpfli, 1975). It is also worth stressing here that the very different estimates for $M_{N a}$ and $M_{K}$ provide further support for the idea that sodium and potassium channels exist as distinct physical entities, in contrast with the single channel hypothesis describing the sodium and potassium currents as temporally separated processes utilizing the same type of membrane site (Mullins, 1959, 1968).

\section{Restrictions on possible channel models}

The simple kinetic scheme used in the $\mathrm{HH}$ equations (Hodgkin \& Huxley, 1952) implies a rather precise picture of the potassium and sodium channels in nerve membranes. Such picture has been assumed in deriving equations (29), (31), (32) and (34), which are consistent with the $\mathrm{HH}$ description of the average relaxation (voltage-clamp) currents, as shown by (30) and (33). It is known, however, that a large variety of different models of channel kinetics may lead to equally adequate descriptions of the voltage-clamp experimental curves (Stevens, 1972; Hill \& Chen, 1972 $a$; Chen \& Hill, 1973). Indeed, in the case of potassium currents, it was even shown that depolarizations from a wide range of holding potentials, including large hyperpolarizations, are much better fitted by exponentials raised to a 25 th, rather than to a 4 th, power (Cole \& Moore, 1960).

These considerations raise two problems concerning the analysis of noise data. First, since the estimates of $M_{K}$ and $M_{N a}$ constitute the only information extracted from noise measurements that is not contained in voltage-clamp data, one should ask how much such estimates depend on the theoretical model used. Second, one may investigate whether the realm of plausible channel models is restricted by the results of noise measurements. For sodium channels these problems are of minor relevance, mainly because estimates of $M_{N a}$ are available which do not depend on particular models of channel kinetics (Keynes et al. 1975; Levinson \& Meves, 1975). Furthermore, the simple $\mathrm{m}^{3} \mathrm{~h}$ kinetic scheme has already received strong support at the microscopic level from the measurements of the displacement currents associated with the movement of three independent channel-activating gates (for reviews see Armstrong, 1975; Keynes, 1975). 
Accurate sodium channel noise measurements might yield valuable independent contributions to the understanding of many problems, concerning the refined kinetics of the $\mathrm{Na}$ channels, which have been raised by gating currents measurements (see Keynes, 1975). However, such a contribution is impossible within the accuracy and the range of present data.

For potassium channels, the above problems require a more detailed discussion. No direct estimates of $M_{K}$ exist, while the observations of Cole \& Moore (1960) raise serious doubts about the validity of the simple $n^{4}$ kinetic scheme adopted by Hodgkin \& Huxley (1952), which is not supported by gating current measurements as in the case of sodium channels. We shall examine in what follows the consequences of interpreting the above reported potassium channel noise data in squid giant axons, according to two generalized $\mathrm{HH}$ type models discussed theoretically by Hill \& Chen (1972a) and Chen \& Hill (1973), or according to a continuous model discussed by Stevens (1972).

The most important considerations, in interpreting noise measurements according to different microscopic models, is that the parameters required for the fitting of noise data should be derived from voltage-clamp experiments according to the same models.

$X$ subunits $H H$ model. The theoretical characterization of this model is given by equations (35) and (36), with $\rho_{n}^{(x)}=\left[\mathrm{I}-\bar{Y}_{n}^{(x)}\right] / \bar{Y}_{n}^{(x)}$ (see equation (I0) for $\kappa=0$ ). For $x=25$ equation (36) yields the kinetic scheme for the description of potassium repolarization adopted by Cole \& Moore (1960).

Following (35) the power spectrum, $S_{K}^{(x)}(f)$, for the potassium channel noise according to this model is given, for arbitrary $x$, by:

$$
\frac{M_{K} S_{K}^{(x)}(f)}{\bar{I}_{K}^{2}}=s_{K}^{(x)}(f)=4 \tau_{n}^{(x)} \sum_{i=1}^{x}\left(\begin{array}{l}
x \\
i
\end{array}\right)\left[\rho_{n}^{(x)}\right]^{i} / i\left[\mathrm{I}+\left(2 \pi f \tau_{n}^{(x)} / i\right)^{2}\right],
$$

where the average current, $\bar{I}_{K}$, should be considered as a purely experimental, fixed, quantity; the function $s_{K}^{(x)}(f)$ represents the single channel noise spectral density relative to the mean single channel current squared; and where $\rho_{n}^{(x)}=\left(\mathrm{I}-\bar{Y}_{n}^{(x)}\right) / \bar{Y}_{n}^{(x)}$ and $\tau_{n}^{(x)}$ should be derived from a fitting of voltage-clamp curves according to equation $\left(3^{6}\right)$.

Figure 7 shows an example of how $\rho_{n}^{(x)}$ and $\tau_{n}^{(x)}$ are derived from a real experiment. The experiment was performed on a giant axon immersed in normal sea water containing $10^{-7} \mathrm{M}$ TTX at $6{ }^{\circ} \mathrm{C}$. The 


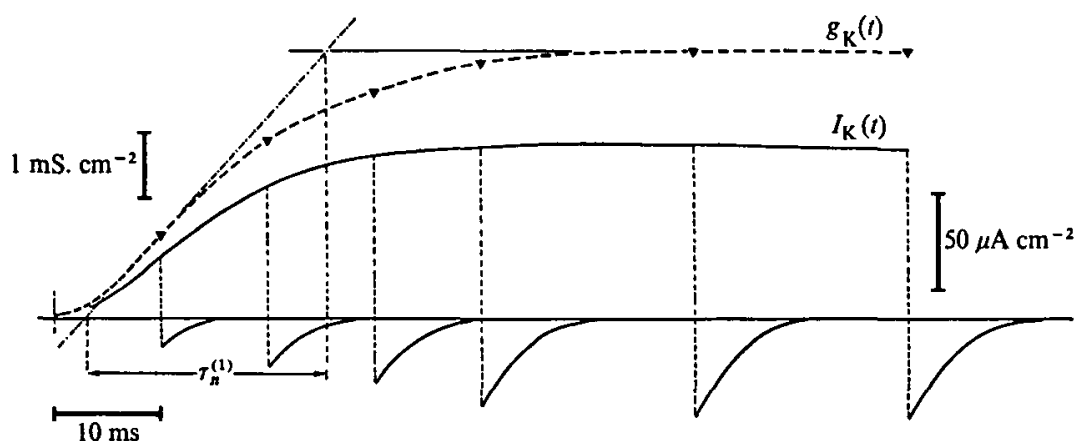

Fig. 7. Time course of the potassium current, $I_{K}(t)$ and potassium conductance, $g_{B}(t)$ in a voltage-clamp experiment on a squid giant axon of Loligo vulgaris. The axon was immersed in normal sea water at $6^{\circ} \mathrm{C}$ in the presence of $100 \mathrm{nM}-\mathrm{TTX}$ and kept at a holding (hyperpolarizing) membrane potential of $-80 \mathrm{mV}$. Square depolarizing voltage pulses of $35 \mathrm{mV}$ amplitude and various durations were then applied, producing the solid line current traces drawn in the figure (where capacitative artifacts have been removed). The time course of the potassium conductance was obtained from the current jumps at the end of the various pulses. The slope of the tangent to $g_{K}(t)$ at the inflection point, and the plateau value of $g_{K}(t)$ are independent of the holding potential. For the use of the time constant, $\tau_{n}^{(1)}$, see text.

axon was normally maintained in space-clamp, voltage-clamp conditions at a holding potential of $-80 \mathrm{mV}$, from which sudden depolarizations to $-45 \mathrm{mV}$ were applied in successive traals with increasing duration. The superposed records of the measured potassium currents (suitably corrected for capacitive artifacts) are shown in Fig. 7 as solid lines. The time course of the potassium conductance, $g_{K}(t)$, derived from the current jumps at the end of each pulse, is also drawn in the figure. This describes the kinetics of potassium channels more accurately than the time course of the current, which is also affected by changes in the potassium equilibrium potential due to potassium ion accumulation in the extracellular space (Frankenhaeuser \& Hodgkin, 1956). From the asymptotic value of $g_{K}, g_{K}(\infty)$, and the maximum potassium conductance, $\bar{g}_{K}$, obtained from similar experiments with large depolarizing voltage steps (to $V_{m}>+50 \mathrm{mV}$ ), $\rho_{n}^{(x)}$ is derived as:

$$
\rho_{n}^{(x)}=\left\{\bar{g}_{K} / g_{K}(\infty)\right\}^{1 / x}-\mathrm{I} \text {. }
$$

Finally, $\tau_{n}^{(x)}$ is obtained from the maximum slope of $g_{K}(t) / g_{K}(\infty), \mathrm{r} / \tau_{n}^{(1)}$, as:

$$
\tau_{n}^{(x)}=\{(x-1) / x\}^{x-1} \cdot \tau_{n}^{(1)} .
$$

It is important to stress that the determination of $\rho_{n}^{(x)}$ and $\tau_{n}^{(x)}$, according to equations (44) and (45), involves only measurements of $g_{K}(\infty)$ and 


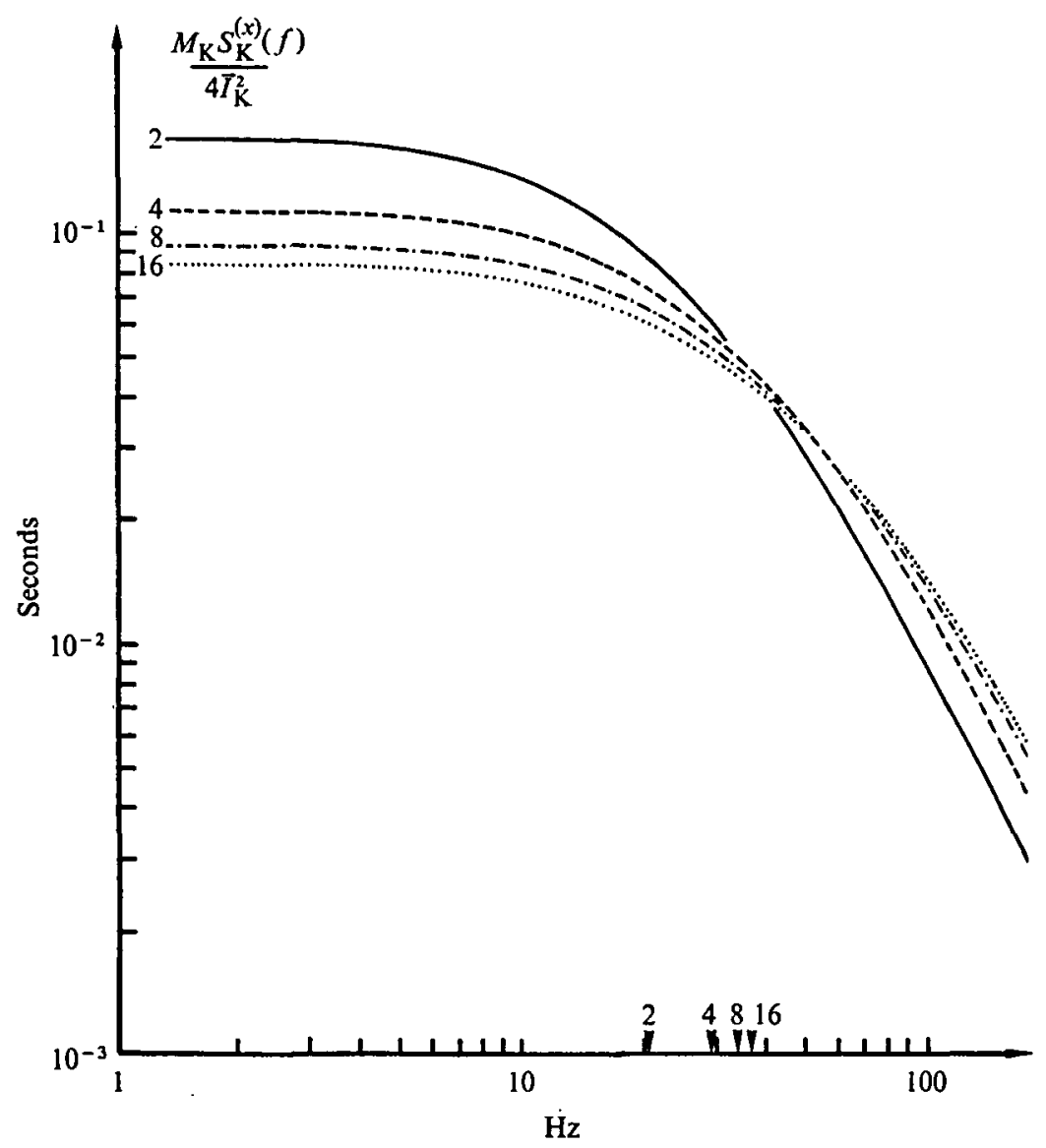

Fig. 8. Log-log plot of the relative current noise spectral density, $s_{k}^{(x)}(f) M_{R} S_{K}^{(x)}(f) / 4 I_{R}^{2}$, of a single $x$ subunit $H H$ potassium channel, for different values of $x$. The curves were obtained using the following parameters, derived from the experiment of Fig. 7:

$$
\begin{array}{llll}
\rho_{n}^{(2)}=3.94 & \rho_{n}^{(4)}=1.22 & \rho_{n}^{(8)}=0.49 & \rho_{n}^{(16)}=0.22 \\
\tau_{n}^{(2)}=11 \mathrm{~ms} & \tau_{n}^{(4)}=9.2 \mathrm{~ms} & \tau_{n}^{(8)}=8.6 \mathrm{~ms} & \tau_{n}^{(16)}=8.4 \mathrm{~ms}
\end{array}
$$

The arrows indicate the half power frequencies of the various spectra.

$\tau_{n}^{(1)}$, which are experimentally independent of the holding potential (Cole \& Moore, ig60; also our observations).

The values of $\tau_{n}^{(x)}$ and $\rho_{n}^{(x)}$ for $V_{m}=-45 \mathrm{mV}$, obtained from the experiment of Fig. 7 , have been used to draw the plots of $s_{K}^{(x)}(f)$, shown in Fig. 8, for $x=2,4,8$ and 16 . Similar data at four different membrane potentials have been used to determine the voltage dependence of $s_{K}^{(x)}(0)$, for the same values of $x$, shown in Fig. 9. These figures contain all the 


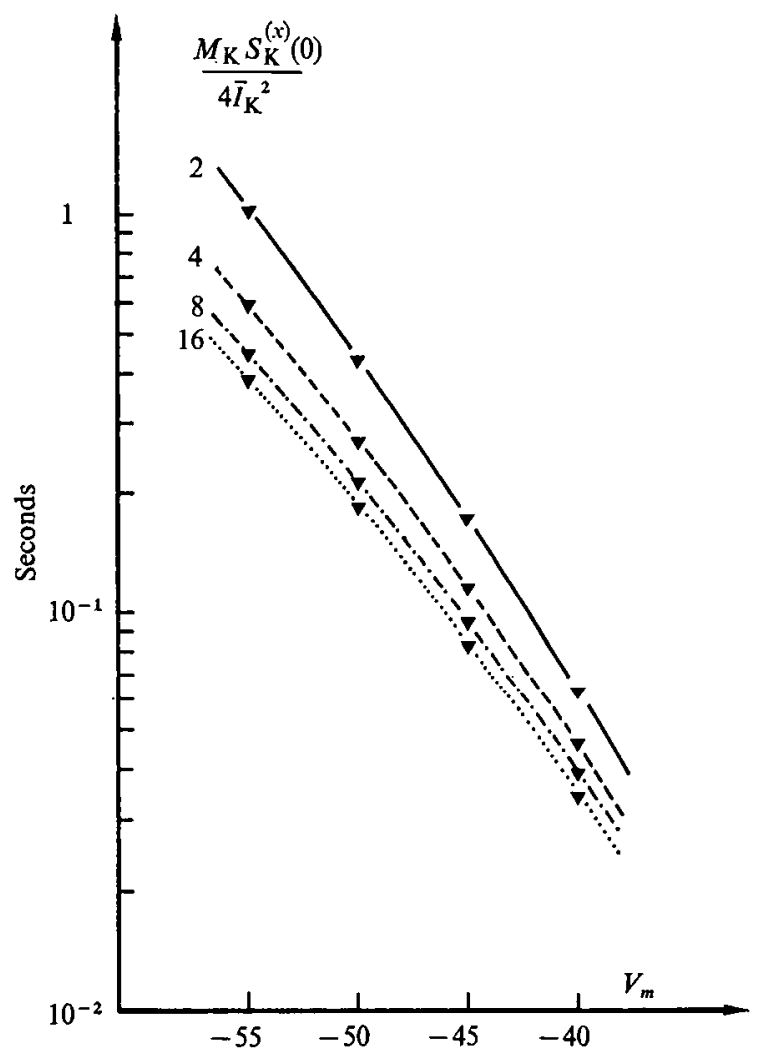

Fig. 9. Plot of $\log s_{E}^{(x)}(0)$ versus membrane potential for the same $x$ values of Fig. 8. The curves were obtained using the values of $\rho_{n}^{(x)}$ and $\tau_{n}^{(x)}$ extracted from four experiments, similar to that of Fig. 7, performed on the same axon with different depolarizing voltage steps $(25,30,35$ and $40 \mathrm{mV})$.

required information to answer the two questions posed at the beginning of this section, for the $x$ subunit HH channel model.

It is seen from Fig. 9 that the expected voltage dependence of $S_{K}^{(x)}(0)$ is fairly independent of $x$. Thus the experimental $S_{K}($ o) data do not indicate whether a particular value of the parameter $x$ is better than another. On the other hand, the estimate of $M_{K}$ is not strongly affected by the choice of $x$. The value $M_{K}=60 \mu \mathrm{m}^{-2}$, obtained by Conti et al. (1975) assuming $x=4$, would become $M_{K} \sim 100 \mu \mathrm{m}^{-2}$ for $x=2$, and $M_{K} \sim 40 \mu \mathrm{m}^{-2}$, for $x=16$.

The measurements of $f_{\frac{1}{2}}^{(K)}$ seem to allow a better discrimination between permissible $x$ values. It is seen from Fig. 8 that the expected value of $f_{\frac{1}{2}}{ }^{(K)}$, at $-45 \mathrm{mV}$ and $6{ }^{\circ} \mathrm{C}$, increases from about $20 \mathrm{~Hz}$ to about 
$3^{8 \mathrm{~Hz}}$ upon increasing $x$ from 2 to 16 . In the measurements of Conti et al. (1975; Fig. 7) $f_{\frac{1}{2}}^{(K)}$ fell in the range between 20 and $40 \mathrm{~Hz}$. More recent results, obtained by the present authors, led to a smaller scattering of $f_{\frac{K}{8}}^{\left(\frac{K}{2}\right)}$ data, which at $-45 \mathrm{mV}$ and $6{ }^{\circ} \mathrm{C}$ ranged in four experiments between 20 and $30 \mathrm{~Hz}$. Thus, these measurements indicate that the $x$ subunit $\mathrm{HH}$ model can adequately fit noise data only for $2 \geqslant x \geqslant 4$. This conclusion could be reconciled with Cole \& Moore's (I960) observations by assuming, as proposed by Hill \& Chen (1972 $b$ ), that hyperpolarization introduces some additional phenomenon which is more faithfully described by a real delay in the start of the normal $\mathrm{HH}$ potassium activation, than by a large value of $x$. Since such phenomenon would become very important only below the resting potential $\left(V_{R} \sim-60 \mathrm{mV}\right)$, and it is not expected to show up in the small fluctuations occurring near or above $V_{R}$. It is interesting that a similar delay seems to occur also in the sodium channels (Keynes \& Rojas, I975) for which the evidence of a small number of subunits is independently supported by measurements of gating currents.

4 subunits ' $K$ case'. Among the possible generalized $\mathrm{HH}$ models, which succeed in predicting average current relaxations compatible with voltage-clamp data, the ' $\kappa$ model' introduced by Hill $\&$ Chen $(1972 a)$ is particularly attractive for its simplicity and physical soundness. The model merely relaxes the condition that an ionic channel is completely impermeable to ions whenever one of its subunits is in the 'closed' state. Rather it assumes that the transition of any subunit from the 'open' to the 'closed' state decreases the channel conductance, e.g. by raising a potential barrier to the passage of ions, by a factor $\kappa^{-1}\{\kappa<I\}$. For potassium channels, the conductance in any given channel configuration is then $\gamma \kappa^{\alpha}$, where $\alpha$ is the number of channel subunits in the closed state. The theoretical characterization of this model is given by equations (35) and $(36)$ and the power spectrum by (43), where

$$
\rho_{n}^{(x)}=\left[\mathrm{I}-\bar{Y}_{n}^{(x)}\right]\left[\bar{Y}_{n}^{(x)}-\kappa\right] /\left[\bar{Y}_{n}^{(x)}\right]^{2} .
$$

Conti et al. (1975) have analysed their noise data according to this model, for $x=4$. They found that only for $\kappa \leqslant 0$. I a fair fitting of the experimental voltage dependence of $S_{K}(0)$ could be obtained. For $\kappa=0 \cdot \mathrm{I}$ the estimate of $M_{K}$ changed from 60 to $40 \mu \mathrm{m}^{-2}$.

Continuous state model. This model was discussed by Stevens (1972), and was indicated by Fishman (1973) as yielding a better fit of the 
cut-off frequencies of 'voltage noise' from small patches of squid axon membrane. It hypothesizes that the conductance of a single potassium channel is given by $\gamma_{K} n^{4}$, where $n$ is a stochastic variable, ranging between 0 and $\mathrm{r}$, which fully describes the statistical properties of the channel. For $\Delta n \ll \bar{n}$, the relaxation of $\gamma_{K} n^{4}$ is linear in $\Delta n$ and, according to the $\mathrm{HH}$ equations, follows an exponential decay with time constant $\tau_{n}$. For this model the fluctuation-dissipation theorem (see Appendix) leads to an expected potassium channel noise power spectrum in the form of a simple Lorentzian with cut-off frequency $\left(2 \pi \tau_{n}\right)^{-1}$ (Stevens, 1972). Quite apart from the general comments of Chen \& Hill (1973) it appears that this model should be discarded because of the following quantitative considerations.

Although it is impossible to obtain a theoretical estimate of the absolute power of the expected noise, an upper limit for it is imposed by the implicit assumption that $\sigma_{n} \ll \bar{n}$, where $\sigma_{n}$ is the standard deviation of the fluctuations of $n$ around its average value $\bar{n}$. If $\Delta g$ indicates the displacement of the single channel conductance from its average value, $\bar{g}$, this assumption implies that:

$$
\left\langle(\Delta g)^{2}\right\rangle / \bar{g}^{2}=\left\langle(4 \Delta n)^{2}\right\rangle / \bar{n}^{2}=\mathrm{I} 6 \sigma_{n}^{2} / \bar{n}^{2}<\sim 0 \cdot 16 .
$$

On the other hand, the simple $\mathrm{HH}$ model predicts for the single channel conductance fluctuations at $V_{m}=-45 \mathrm{mV}$ (equation (29) for $M=\mathrm{I}$ ):

$$
\left\langle(\Delta g)^{2}\right\rangle / \bar{g}^{2}=\phi_{I}(0) / \bar{I}^{2}=\left(\mathrm{I}+\rho_{n}\right)^{4}-\mathrm{I} \sim 20,
$$

where the value $\rho_{n}=\mathrm{I} \cdot 22$ is obtained from the experiment of Fig. 7 . Even larger values of $\left\langle[\Delta g]^{2}\right\rangle \mid \bar{g}^{2}$ would result from values of $\rho_{n}$ at smaller depolarizations. Comparison of equations (47) and (48) shows that the continuous model would require that the density of the potassium channels is at least two orders of magnitude lower than that estimated by Conti et al. (I975). These channels, more than I $\mu \mathrm{m}$ apart from each other, would have a conductance in the open state much larger than I $\mathrm{nS}$, two orders of magnitude higher than that of the non-selective gramicidin A channels in artificial lipid bilayers (Hladky \& Haydon, I970, 1972).

\section{NOISE FROM LIPID BILAYERS}

It is well known that artificial lipid bilayers (BLM), although being intrinsically almost impermeable to ions, can acquire electrical con- 
ductances comparable to those of natural biological membranes in the presence of a large number of substances providing extrinsic ionic pathways across their hydrocarbon region (Mueller et al. 1962; for a comprehensive treatise of the basic properties of BLM systems, see e.g. Fettiplace et al. I975). These substances seem to operate following two basically distinct physical mechanisms. A carrier mediated transport mechanism, in which ions are solubilized in the lipid phase by forming complexes with large liposoluble molecules, describes quite accurately the effect on lipid bilayers of macrocyclic antibiotics, such as valinomycin and nonactin (for a review see e.g. Laüger, 1972). On the other hand, the very low ionic selectivity and the current voltage characteristics of BLM incorporating monazomycin (Muller \& Finkelstein, 1972a, 1972 $b$ ) or the polyene antibiotics Nystatin and Amphotericin B (Cass, Finkelstein \& Crespi, 1970; Finkelstein \& Holz, 1972) suggest that these substances form real pores across the lipid structure.

Perhaps the most unequivocal characterization of a pore mechanism as opposed to a carrier mediated one, is obtained in terms of the conductance of a single elementary ionic pathway. In fact the upper limit of about $10^{4} \mathrm{~s}^{-1}$, for the turnover of a carrier such as a nonactin molecule (Laüger, 1972), places an upper boundary of the order of $10^{-14} \mathrm{~S}$ to the conductance of a single channel operating with such a mechanism. Thus the measurements, in BLM systems incorporating EIM, gramicidin A, alamethicin, nystatin and monazomycin, of single channel conductances which are several orders of magnitude higher than the above figure, confirm other less direct evidence that these substances act as pores. All single channel conductance estimates in BLM are based on the measurements of channel conductance fluctuations reviewed below. Of course, the importance of such measurements is not limited to the possibility of discriminating pores versus mobile carriers. They provide also basic information about the kinetic properties of the ionic channels, which is not contained in measurements of average steady state currents and may be difficult to extract from relaxation measurements.

Measurements of channel conductance fluctuations have been mostly performed on BLM systems containing (either semi-permanently or on the average) just one or very few ionic channels (Bean et al. 1969; Ehrenstein, Lecar \& Nossal, 1970; Hladky \& Haydon, 1970, 1972; Gordon \& Haydon, 1972; Haydon, Hladky \& Gordon, 1972; Latorre, Ehrenstein \& Lecar, 1972; Bean, 1972; Eisenberg, Hall \& Mead, I973; 
Boheim, I974; Ehrenstein etal. 1974; Alvarez, Latorre \& Vergudo, 1975. Gordon \& Haydon, 1975). The application of the more general type of noise analysis discussed in this review, to BLM systems containing a large number of channels, is relatively more recent (Zingsheim \& Neher, 1974; Neher \& Zingsheim, 1974; Kolb et al. 1975; Wanke, 1975) We shall discuss these two types of measurements separately.

\section{Single channel conductance fluctuations}

EIM. EIM (excitability inducing material) is a still uncharacterized proteinaceous substance which confers large ionic conductances and excitability properties on lipid bilayers (Mueller \& Rudin, 1963). As first realized by Bean et al. (1969), the addition to one of the two membrane bathing solutions of trace amounts of EIM, produces discrete progressive conductance increments attributed by the authors to the incorporation in the membrane of an increasing number of individual EIM molecules. Later studies by Ehrenstein et al. (1970) confirmed that in oxidized cholesterol membranes, EIM forms individual ionic channels with lifetimes of many minutes, which can exist in two different conductance states with probabilities dependent on membrane voltage. Although it has been shown that in BLM with different composition the EIM channels may have more than two states (Bean, 1972), we shall discuss only the case of oxidized cholesterol membranes which is simpler and better studied.

All the experimental evidence (Ehrenstein et al. 1970, 1972, 1974; Latorre et al. 1972; Alvarez et al. 1975) indicates that the EIM channel in oxidized cholesterol membranes is fully characterized by its two conductance levels, $\gamma_{c}$ and $\gamma_{0}$, and by the two rate constants $\alpha$ and $\beta$ which determine its stochastic behaviour according to equations (2), (3) and (4). It was found that $\gamma_{c}$ and $\gamma_{0}$ do not depend on voltage, while they are changed by altering the concentration of monovalent cations in the bathing solutions. The selectivity among different monovalent cations, either measured by the ratios of $\gamma_{0}$ or by the permeability ratios derived from bionic potentials, was found to follow the scale of the cation mobilities in free solution, suggesting that the channels are wide pores $(\sim \mathrm{I} 6 \AA$ in diameter) containing inside a negative electronic charge (Latorre et al. 1972). Typical reported values for $\gamma_{0}$ and $\gamma_{c}$ in $0.1 \mathrm{MV}-\mathrm{KCl}$ are: $\gamma_{0}=0.4 \mathrm{nS} ; \gamma_{c}=0.08 \mathrm{nS}$. The rate constants $\alpha$ and $\beta$ were found to be exponential functions of membrane potential, and led to a correct estimate of the time constant, and of the percentage channel 
activation measured from voltage-clamp experiments on membranes containing a large number of channels (Ehrenstein et al. 1974). The agreement between single channel kinetics and macroscopic relaxation properties is maintained also when $\alpha$ and $\beta$ are changed by altering the temperature or the lipid composition of the membranes (Alvarez et al. 1975).

The results summarized above show that the similarity between the EIM channels in BLM and the ionic channels in nerve membranes goes beyond the simple phenomenological analogy of the excitability properties of the two systems. Each EIM molecule behaves very similarly to the individual subunits postulated in sodium or potassium $\mathrm{HH}$ channels. A major difference consists in the absolute value of $\tau=(\alpha+\beta)^{-1}$, which is one or two orders of magnitude higher for EIM channels. However, this difference is not a qualitative one, and it might merely indicate that the 'gate' of EIM channels has to overcome a higher energy barrier than its equivalent structures in the nerve membranes. It is a real pity that the lack of characterization of EIM in terms of molecular structure makes this gating mechanism not better understood than those operating in nerves.

Alamethicin. Elementary conductance changes in BLM containing alamethicin have been studied by various authors (Gordon \& Haydon, 1972; Haydon et al. 1972; Eisenberg et al. 1973; Boheim, 1974; Gordon \& Haydon, 1975). The alamethicin channels appear from these studies to be far more complicated than EIM channels. Thus the unitary event associated with the appearance of a channel at a constant membrane voltage is a train of many discontinuous jumps between as many as 9 (according to Gordon \& Haydon, 1975) different current levels. A channel state of zero conductance, which at low membrane potentials has much higher probability of occurrence than any of the non-zero conductance states, seems to exist as distinct from the situation of zero current in the absence of any channel. The conducting states of alamethicin channels have very high ionic permeabilities. The various conductance levels in $0.5 \mathrm{M}-\mathrm{NaCl}$ vary from about $0.6 \mathrm{nS}$ to about $10 \mathrm{nS}$, and are independent of the membrane thickness which affects, however, the frequency of opening and the average lifetime of the channels (Gordon \& Haydon, 1972; Haydon et al. 1972). At all levels of conductance, the channels show very little discrimination between cations and anions or even between mono- and divalent ions, the ratios of the conductances for univalent cations being close to those in aqueous 
solution (Haydon et al. 1972). All these results provided strong evidence for identifying the alamethicin channels as long-lived wide pores, whose structure is little affected by changes in the host lipid membrane matrix.

The most interesting feature of the electric conductance of lipid bilayers containing alamethicin is its very strong voltage dependence. Studies of single channel fluctuations have identified this voltage dependence as being mainly confined to the probability of leaving the zero conductance state, while the transition probabilities between different conducting states are comparatively little affected by voltage (Eisenberg et al. 1973; Boheim, I974; Gordon \& Haydon, 1975). This result is in agreement with voltage-clamp data (Mauro, Nanavati \& Heyer, 1972; Eisenberg et al. r973) showing essentially a single exponential relaxation of the conductance in BLM containing a large average number of alamethicin channels. It is in fact expected that the voltage steps should mainly alter the relative population of channels in the zero conductance state while they should produce little redistributions within the probabilities of the conducting states. These redistributions would account for the initial inductive behaviour of the voltage-clamp currents which occurs on a time scale comparable with the average lifetime of the various conducting states.

The physical interpretation at the molecular level of the various states of alamethicin channels is still debated. The dependence of the average membrane conductance on the gth power of the alamethicin concentration indicates that oligomers containing up to 9 alamethicin molecules maybeinvolved in the formation of a single channel. However, according to Boheim (1974) this does not imply that only complexes of 9 molecules can form a channel. Instead, it may be that oligomers containing from 2 to 9 molecules are associated with channel states of increasing conductance, the dimer configuration corresponding to the zero conductance state of a formed channel. In support of this interpretation (which, incidentally, implies only 7 non-zero conductance levels), it is estimated that the diameter of a ring-shaped oligomeric pore would increase by addition of monomers in a way which is consistent with the increase in the various conductance levels. Apart from other evidence against the hypothesis of an increasing pore diameter (Gordon \& Haydon, 1975), it is difficult to understand in this model why the rate constants involved in the formation and disappearance of the dimer state should be orders of magnitude smaller than those involved in the successive growing of the channel. It seems also odd that the transition from 
a dimer to a trimer should be far more voltage dependent than the further growth of the channel.

In the model proposed by Gordon \& Haydon (1975) only oligomers containing 9 alamethicin molecules can form a channel. Such complexes can exist in a non-conductive state (e.g. when merely adsorbed at one membrane solution interface) or in a conductive state (when more deeply embedded in the membrane), the latter configuration favouring the possible successive transformation of each monomer in a direct channel spanning across the membrane. The actual opening of these channel subunits requires a last step (e.g. the removal of a residual layer of lipids on the side opposite to the penetration of the complex) whose probability is fairly independent of membrane potential. In this model the membrane potential mainly affects the penetration of the formed complex into the membrane. By assuming that this occurs through the interaction of the electric dipole moment of the complex with the applied electric field one can also account qualitatively for the asymmetric properties of alamethicin channels upon reversal of the membrane potential. The evidence in support of the parallel functioning of many monomeric channels is based on selectivity data, showing an abrupt cut-off of the permeability for cations of certain ionic radius, independently of the channel conductance state (Gordon \& Haydon, 1975). However, the physical reason why only a complex of 9 alamethicin molecules should be able to flip to a conductive state, which only involves monomeric pores, is not clear. Furthermore, this model fails to account for the dependence on alamethicin concentration of the fast rebound ( $50 \mu \mathrm{s} \div \mathrm{I} \mathrm{ms}$ ) of voltage-clamp currents (Mauro et al. 1972). In fact the only time constants which are expected to depend on the alamethicin concentration are the very slow ones governing the formation of the large complexes or the adsorption of monomers at the membranesolution interface. A qualitatively correct concentration dependence of the fast rebound would be expected, however, if the transitions between the various conductance states of the same channel involved oligomermonomer reactions, as in the first model discussed above.

Thus, it appears that the best physical description of the alamethicin channels should be found in a compromise between the two proposed models. A careful study, of the single channel fluctuations or of the noise from many channels, as a function of alamethicin concentration could yield very useful information in this respect, as it did in the case of the gramicidin A channels discussed below. 
Gramicidin $A$. Black lipid membranes containing the linear polypeptide gramicidin A owe their high electrical conductance to the formation by the antibiotic molecules of porous structures spanning across the bilayer. The best experimental evidence of these pores was provided by single channel kinetic studies (Hladky \& Haydon, I970, 1972). The essential results of these works are summarized below.

In the presence of trace amounts of gramicidin, lipid bilayers of various compositions show discrete, randomly occurring conductance steps of approximately constant amplitude. When multiple steps are observed, their amplitude distribution is Poissonian, indicating that they derive from the contribution of statistically independent unitary steps. The unitary conductance change, for a constant ionic composition of the bathing solutions, is fairly independent of membrane thickness (varied by changing the membrane composition) and of temperature $\left(Q_{10} \simeq 1 \cdot 4\right)$. This elementary event is then attributed to the creation of a single pore whose internal structure is not much influenced by the host membrane matrix. The pore conductance, fairly ohmic, has typical values of $2 \times 10^{-11} \mathrm{~S}$ and $4.8 \times 10^{-11} \mathrm{~S}$ in $\mathrm{I} \mathrm{M-NaCl}$ and $\mathrm{I} \mathrm{M}-\mathrm{KCl}$, respectively. On the other hand, the average value of the exponential distribution of the pores' lifetime is strongly dependent of membrane thickness and temperature $\left(Q_{10} \simeq 3\right)$, in agreement with the idea that the anchorage between two gramicidin A molecules from opposite membrane sides is responsible for the creation of the pore (Urry, I97I; Urry et al. 1971). More precisely, according to Hladky \& Haydon (1972), the quantitative data are only consistent with the special model of such a dimeric channel proposed by Urry et al. (197I).

The strongest direct evidence for the dimeric structure of the gramicidin A channels was obtained from voltage-clamp relaxation measurements (Bamberg \& Laüger, I973). These measurements exploited the significant dependence of the average number of channels upon the voltage applied across the membrane, probably due to electrostriction (Hladky \& Haydon, 1972). It was found that the current relaxation following a voltage step had a simple exponential time course, with a time constant, $\tau$, which depended on the amount of gramicidin A present. Assuming that the formation of gramicidin A channels is governed by the reaction:

$$
\mathrm{G}+\mathrm{G} \underset{K_{D}}{\stackrel{K B}{\rightleftarrows}} \mathrm{G}_{2}
$$


where $G$ and $G_{2}$ represent a monomer and a dimer, respectively, one expects that $\mathrm{I} / \tau$ varies linearly with the average concentration of membrane adsorbed monomers, $N_{m}$ (Bamberg \& Läuger, 1973). Furthermore, still according to equation (49), $N_{m}$ is expected to be proportional to the square root of the average dimer concentration, $N_{d}$, which determines the average current, $I$, flowing through the membrane. For a fixed final voltage, the plot of $\mathrm{I} / \tau$ versus $\sqrt{ } I$ was actually found to lie on a straight line, whose slope and intercept to the origin allowed to evaluate the rate constants, $K_{D}$ and $K_{R}$, in equation (49). The values for $K_{D}$ agreed fairly well with the reciprocal of the average single channels life time measured by Hladky \& Haydon (1972). In contrast to $K_{D}, K_{R}$ was found to be strongly voltage dependent, in qualitative agreement with the observations on few channels, although the latter did not allow an absolute estimate of $K_{R}$. It should be stressed, on the other hand, that the evaluation of $K_{R}$ from relaxation experiments required the previous knowledge of the channel conductance, $\Lambda$, obtained from single channel kinetic observations. It may be interesting to report here the estimate for the equilibrium constant of the reaction (49), $K=K_{R} / K_{D} \sim 2 \times 10^{14} \mathrm{~cm}^{2} \mathrm{M}^{-1}\left(V_{m}=125 \mathrm{mV}\right.$ ) (Bamberg \& Läuger, 1973). According to this figure, membrane areas as small as those used by Hladky \& Haydon $(1972)\left(3 \times 10^{-5} \mathrm{~cm}^{2}\right)$, contain on the average only one open channel (dimer) in the presence of 300 monomers.

\section{Noise in the presence of many channels}

Detailed studies of noise from a large number of channels have been concerned so far only with lipid bilayers containing gramicidin A (Neher \& Zingsheim, I974; Zingsheim \& Neher, 1974; Kolb et al. 1975). The results of these works did not add new information to what had been obtained from the single channel studies referred to above. Their main relevance consists, therefore, in showing the validity of fluctuation analysis for studies of biological membranes, the choice of an already fully described system being most appropriate to this purpose. Thus, it was shown that all the parameters required for the characterization of the gramicidin A channels could be measured directly from the autocorrelation function of the membrane current noise $\phi_{I}(t)$. As expected from the fluctuation-dissipation theorem (see Appendix) and from the linear relaxation properties of the system (Bamberg \& Läuger, 1973), $\phi_{I}(t)$ is found to follow a simple exponential decay with time constant $\tau$. As for the analysis of the relaxation measurements (Bamberg \& Läuger, 
1973) the plot of $I / \tau$ as a function of the square root of the average membrane conductance leads to the determination of the rate constants, $K_{D}$ and $K_{R}$ in equation (49). However, while the evaluation of $K_{R}$ from voltage-clamp data relies upon an independent estimate of the single channel conductance, $\Lambda$, (Bamberg \& Läuger, 1973) noise data are completely self-consistent, since $\Lambda$ can be evaluated from the low conductance limit of $\phi_{I}(0)$ (Neher $\&$ Zingsheim, 1974; Zingsheim \& Neher, 1974; Kolb et al. 1975). All the values of $K_{D}, K_{R}$ and $\Lambda$ estimated from noise analysis were found to be in very good agreement with those reported by Hladky \& Haydon (1972) and by Bamberg \& Läuger (1973).

Very recently, observations of channel noise in lipid bilayers containing monazomycin and nystatin have also been reported (Wanke, r975). The results are still preliminary and have not yet been analysed quantitatively. They merely show that the noise power of the current fluctuations induced by these substances is far larger than that expected from channels operating with a carrier mechanism, confirming the idea, supported also by other evidence (Muller \& Finkelstein, 1972 $a, 1972 b$; Cass et al. 1970; Finkelstein \& Holz, 1972), that monazomycin and nystatin form real pores across the lipid structure. The estimates of pore conductance given by Wanke (1975) $\left(5 \times 10^{-14} \mathrm{~S}\right)$ for monazomycin in $0.1 \mathrm{M}-\mathrm{KCl} ; 6 \times 10^{-10} \mathrm{~S}$ for nystatin in $0.2 \mathrm{M}-\mathrm{NaCl}$ ), based on a simple model of two channel conductance states of equal probabilities, should be considered purely indicative, since the choice of the correct model, which should be also consistent with voltage-clamp data, may be very critical for such estimates. These results indicate, however, that noise analysis is at present the most powerful tool for investigating ionic pores whose conductances are not large enough to allow single channel kinetic studies.

\section{APPENDIX}

We give below a brief outline of the basic concepts and theoretical results underlying the analysis and the interpretation of random signals. Exhaustive treatments can be found in any text-book on stochastic processes and random signal analysis (e.g. Papoulis, 1965; Middleton, 1962; Lee, 1960). The last section of this appendix is devoted to a short discussion of the most common sources of electrical noise. This subject is treated most exhaustively by Davenport \& Root (1958) and Van der Ziel (1970).

Downloaded from https:/www.cambridge.org/core. University of Basel Library, on 30 May 2017 at 21:16:23, subject to the Cambridge Core terms of use, available at https:/www.cambridge.org/core/terms. 


\section{Stochastic variables}

Physically, a random signal is the time course of any observable, $X$, whose value is determined by the random occurrence of a set of possible states of a physical system, which are compatible with a particular average state defining the conditions of the observation. Mathematically, the random occurrence of the various states of the set is described as a stochastic process and the assignment of an $X$ value to each system state characterizes $X$ as a stochastic variable. As such, $X$ is completely specified by the knowledge of the multivariate probability densities, $f_{n}\left[\left(x_{1}, t_{1}\right) ;\left(x_{2}, t_{2}\right) ; \ldots\left(x_{n}, t_{n}\right)\right]$, for any arbitrary integer $n$. Where $f_{n}\left[\left(x_{1}, t_{1}\right) ; \ldots ;\left(x_{n}, t_{n}\right)\right] \mathrm{d} x_{1} \ldots \mathrm{d} x_{n}$ yields the probability of finding $X$ values between $x_{1}$ and $x_{1}+\mathrm{d} x_{1}, \ldots, x_{n}$ and $x_{n}+\mathrm{d} x_{n}$, at times $t_{1}, \ldots, t_{n}$, respectively. $\dagger$

For systems at equilibrium or in a steady state the stochastic process underlying the fluctuations of $X$ will be stationary, implying that:

$$
f_{n}\left[\left(x_{1}, t_{1}+T\right) ; \ldots ;\left(x_{n}, t_{n}+T\right)\right]=f_{n}\left[\left(x_{1}, t_{1}\right) ; \ldots\left(x_{n}, t_{n}\right)\right] .
$$

In what follows, we shall always implicitly assume that we are dealing with stationary stochastic variables.

\section{Expectation values and covariance}

The expectation value, $\langle\Psi\rangle$, of any function, $\Psi\left[X\left(t_{1}\right), \ldots, X\left(t_{n}\right)\right]$, of the stochastic variable $X$, is defined as:

$$
\langle\Psi\rangle=\int_{-\infty}^{\infty} \underset{d}{\mathrm{~d}} x_{1} \int_{-\infty}^{\infty} \mathrm{d} x_{2} \ldots \int_{-\infty}^{\infty} \mathrm{d} x_{n} \Psi\left[x_{1}, x_{2}, \ldots, x_{n}\right] \cdot f_{n}\left[\left(x_{1}, t_{1}\right),\left(x_{2}, t_{2}\right) \ldots\left(x_{n}, t_{n}\right)\right]
$$

Expectation values provide the most direct connection between the mathematical description of a stochastic variable and experiments, since they must be approached by the equivalent statistical averages when the statistics are performed on a large number of trials. In practice the experimental characterization of a stochastic variable is in most cases limited to the evaluation of particular statistical averages associated with the moments of $X$. The moment of order $k$ is defined as:

$\uparrow$ The use of probability densities is most appropriate for the case of continuous variable. However, also variables which can assume only a discrete set of values may be described by generalized probability densities including Dirac $\delta$ functions. 


$$
m_{k}\left(t_{1}, \ldots, t_{k}\right)=\left\langle X\left(t_{1}\right) \cdot X\left(t_{2}\right) \ldots X\left(t_{k}\right)\right\rangle .
$$

The first order moment is the mean of $X$ :

$$
m_{1}=\langle X\rangle=\int_{-\infty}^{\infty} x f_{1}(x) \mathrm{d} x .
$$

The moments of the variable $(X-\langle X\rangle)$ are called central moments of $X$. The first non trivial central moment is that of the second order, called the covariance of $X \phi_{X}(t)$ :

$$
\phi_{X}(t)=\int_{-\infty}^{\infty} \int_{-\infty}^{\infty}\left(x_{1}-m_{1}\right)\left(x_{2}-m_{1}\right) f_{2}\left[\left(x_{1}, t_{1}\right) ;\left(x_{2}, t_{1}+t\right)\right] \mathrm{d} x_{1}, \mathrm{~d} x_{2} .\left(\mathrm{A}_{5}\right)
$$

One of the fundamental properties of the covariance, $\phi_{X}(t)$, is that it is sufficient, together with $\langle X\rangle$, to completely characterize any gaussian random process (see, e.g. ch. 8 of Davenport \& Root, 1958). This is indeed the most frequently encountered type of stochastic process in physical systems, where the random fluctuations of an observed macroscopic variable usually derive from the contribution of a large number of microscopic random events. The gaussian approximation is then legitimized by the central limit theorem (see e.g. Khinchin, 1949).

We state below, without demonstration, some basic mathematical properties of $\phi_{X}(t)$ :

(1) $\phi_{X}(t)=\phi_{X}(-t)=\langle X(t) X(0)\rangle-\langle X\rangle^{2}$.

(2) $\left|\phi_{X}(t)\right| \leqslant \phi_{X}(0)=\left\langle\left(X-m_{1}\right)^{2}\right\rangle$.

(3) Let $X$ be the sum of $N$ independent stochastic variables,

Then

$$
X=\sum_{i=1}^{N} X_{i}
$$

$$
\phi_{X}(t)=\sum_{i=1}^{N} \phi_{X_{i}}(t)
$$

When all the $X_{i}$ have the same covariance, $\phi(t)$ :

$$
\phi_{X}(t)=N \phi(t)
$$

(4) Let $X$ be the product of $N$ independent variables, $X=\prod_{i=1}^{N} X_{i}$, Then

$$
\phi_{X}(t)=\prod_{i=1}^{N}\left\{\phi_{X_{i}}(t)+\left\langle x_{i}\right\rangle^{2}\right\}-\prod_{i=1}^{N}\left\langle X_{i}\right\rangle^{2}
$$




\section{Fluctuation-dissipation theorem}

Many of the theoretical approaches to noise problems try to correlate the properties of the spontaneous fluctuations of an observable to those of its average relaxation following an initial displacement of the system from its final equilibrium state. A general type of fluctuation-dissipation theorem, relating fluctuations and relaxation properties of macrovariables is applicable to a large variety of thermodynamic systems at equilibrium or in a steady state (Kubo, I957; Lax, I960; Kubo et al. 1973). We shall try below to state the essential meaning of such theorem, with particular emphasis on the conditions of its applicability.

Let $X$ be a stationary stochastic observable and let $f_{2}\left[\left(x_{0}, 0\right),(x, t)\right]$ be its second order probability density function. We can always write:

$$
f_{2}\left[\left(x_{0}, \circ\right),(x, t)\right]=f_{1}\left[x_{0}\right] \cdot f_{2}\left[(x, t) /\left(x_{0}, \text { o }\right)\right],
$$

where $f_{2}\left[(x, t) /\left(x_{0}, 0\right)\right]$, defined by (A ro), represents the conditional probability density of finding the value $x$ at time $t$ provided the value $x_{0}$ is observed at time zero. If we confine ourselves to the observation of only those situations for which $X$ assumes the value $x_{0}$ at time zero, the average evolution of our observable for $t>0$ will be given by:

$$
\langle X(t)\rangle_{x_{0}}=\int_{-\infty}^{\infty} x f_{2}\left[(x, t) /\left(x_{0}, \text { o }\right)\right] \mathrm{d} x .
$$

Let us assume that, for all possible initial states with $X=x_{0}$ :

$$
\langle X(t)\rangle_{x_{0}}-\langle X\rangle=\left(x_{0}-\langle X\rangle\right) \mathrm{e}^{-t / \tau}
$$

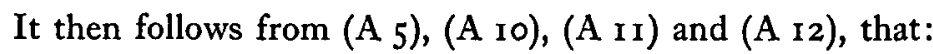

$$
\phi_{x}(t)=\int\left(x_{0}-\langle X\rangle\right) \cdot\left(\langle X(t)\rangle_{x_{0}}-\langle X\rangle\right) \cdot f\left(x_{0}\right) \mathrm{d} x_{0}=\left\langle\left(x_{0}-\langle X\rangle\right)^{2}\right\rangle \mathrm{e}^{-t / \tau}
$$

Equation A I 3 shows that the covariance of the fluctuations of a variable obeying a simple exponential relaxation law is also a simple exponential with the same time constant.

The practical applicability of the equivalence between $\langle X(t)\rangle_{x_{\theta}}$ and $\phi_{X}(t)$ relies on a very critical assumption, which is worth stressing more explicitly. The average relaxation properties of our observable, $X$, can be measured experimentally by following its actual time course, $x(t)$, from an initial value $x_{0}$ which differs from $\langle X\rangle$ much more than in any likely spontaneous fluctuation. Such an initial displacement from $\langle X\rangle$ will be produced by applying appropriate constraints to the system, 
and suddenly removing them at time zero. The observation of a simple exponential relaxation in such experimental conditions is not in general sufficient, however, to demonstrate the validity of the assumptions underlying the derivation of equation ( $\mathrm{A}_{\mathrm{I}_{3}}$ ). It is very critical to show that all possible states with $X=x_{0}$, accessible by spontaneous fluctuations, are also equally well represented in the population of initial states compatible with the applied constraints. For example, it may be that the complete specification of the state of the system requires additional macroscopic variables, $Y_{1}, Y_{2}, \ldots, Y_{n}$, besides $X$, all undergoing independent fluctuations on time scales larger than those characterizing our observations (Lax, 1960). In this case the linear relaxation of the whole set of $(n+I)$ variables will be in general described by the sum of $(n+1)$ exponentials with different time constants. Even if $X$ itself happens to relax (in particular conditions) with a single time constant, $\phi_{X}(t)$ will generally contain a superposition of $(n+r)$ exponentials, with coefficients which can only be evaluated from the kinetic behaviour of the complete set of variables.

\section{Statistical analysis of random signals}

We have already stated that the experimental counterpart of the expectation values, of any function of the time course of a stochastic variable, are the statistical averages over the empirical outcome of a large number of trials. Thus, in principle, the experimental determination of, e.g. $\phi_{X}(t)$ should derive from many independent observations of the time course of the observable $X$, performed either on a large number (ensemble) of statistically identical systems (wherefrom the term 'ensemble average', widely used in statistical mechanics) or on the same system (supposed to be in a stationary state) with large intervals of time between successive observations. In practice the statistical analysis of a random signal is considerably simplified by the assumption that the underlying stochastic process is ergodic. Such a condition can be best understood, in the absence of any rigorous background in probability theory, in terms of its most important practical consequence, namely the equivalence between expectation values and time averages (Birkhoff theorem). For an ergodic stochastic variable $X$ :

$$
\begin{aligned}
& \left\langle\Psi\left[X\left(t_{1}\right), X\left(t_{2}\right), \ldots, X(t n)\right]\right\rangle \\
& \quad=\lim _{T \rightarrow \infty} \frac{\mathbf{I}}{T} \int_{0}^{x} \Psi\left[x\left(t_{1}+t^{\prime}\right), x\left(t_{2}+t^{\prime}\right), \ldots, x\left(t_{n}+t^{\prime}\right)\right] \mathrm{d} t^{\prime},
\end{aligned}
$$


where $x(t)$ is any (possible) outcome of a single observation of the time course of the stochastic variable $X$. In particular, the mean and the covariance of $X$ are measured by the following time averages:

$$
\begin{gathered}
\langle X\rangle=\lim _{T \rightarrow \infty} \frac{\mathrm{I}}{T} \int_{0}^{T} x\left(t^{\prime}\right) \mathrm{d} t^{\prime}, \\
\phi_{x}(t)=\lim _{T \rightarrow \infty} \frac{\mathrm{I}}{T} \int_{0}^{T}\left[x\left(t^{\prime}\right)-\langle X\rangle\right] \cdot\left[x\left(t^{\prime}+t\right)-\langle X\rangle\right] \mathrm{d} t^{\prime} .
\end{gathered}
$$

The time average in the right-hand side of (A I6) is usually called the autocorrelation function of the signal $x(t)$.

One of the most important consequences of the ergodic hypothesis is that the techniques of Fourier analysis of time signals can be exploited for studying random variables. The application is not straightforward, since classical Fourier analysis concerns only periodic or transient signals. However, a formidable generalization to include continuative random signals was obtained by Wiener (for this topic see, in particular, Lee, I960). We state below a major result of such generalization in the form which is widely used in noise analysis. Let $X_{T}(\omega)$ be the truncated Fourier transform of a random signal $x(t)$, with zero mean, defined as:

$$
X_{T}(\omega)=\frac{\mathrm{I}}{2 \pi} \int_{0}^{T} x(t) \mathrm{e}^{-j \omega t} \mathrm{~d} t .
$$

Let $W_{X}$ be the average power of $x(t)$, defined as:

$$
W_{X}=\lim _{T \rightarrow \infty} \frac{\mathrm{I}}{T} \int_{0}^{T} x^{2}(t) \mathrm{d} t .
$$

It can be shown that:

$$
\lim _{T \rightarrow \infty} \int_{-\infty}^{+\infty} \frac{\left|X_{T}(\omega)\right|^{2}}{T} \mathrm{~d} \omega=W_{X}=\phi_{X}(0)=\int_{-\infty}^{+\infty} \Phi_{X}(\omega) \mathrm{d} \omega,
$$

where $\Phi_{X}(\omega)$ is the Fourier transform of the autocorrelation function $\phi_{X}(t)$ of $x(t)$ and the equality between $W_{X}$ and $\phi_{X}(0)$ is a direct consequence of the definitions, equations (A I8) and (A I6). $\Phi_{X}(\omega)$ is called the power spectrum of $X$ (or of our signal $x(t)$ ). Equation (A I9) does not imply the convergence of $\lim _{T \rightarrow \infty}\left|X_{T}(\omega)\right|^{2} / T$. In practice, however, such convergence is not strictly required, since only the total power within a finite (although arbitrarily small) frequency interval is measured. Thus, for all practical purposes, it can be stated that: 


$$
\lim _{T \rightarrow \infty} \frac{\left|X_{T}(\omega)\right|^{2}}{T}=\Phi_{X}(\omega)=\frac{\mathrm{I}}{\pi} \int_{0}^{\infty} \phi_{X}(t) \cos \omega t \mathrm{~d} t .
$$

Equation (A 20) is known as the Wiener-Khinchin theorem, the last equality being a direct consequence of the parity property of $\phi_{X}(t)$, equation (A 6). $\Phi_{X}(\omega)$ is measured in units of $[X]^{2} \mathrm{rad}^{-1}$. Often a power spectrum, $S_{X}(t)$, in units $[X]^{2}$.s, and with the positive and negative frequency contributions lumped together is used.|Since $\Phi_{X}(\omega)=\Phi_{X}(-\omega)$ :

$$
S_{X}(f)=2 \pi\left[\Phi_{X}(\omega)+\Phi_{X}(-\omega)\right]=4 \int_{0}^{\infty} \phi_{X}(t) \cos 2 \pi f t \mathrm{~d} t
$$

From the practical point of view (A 20) implies that random signals can be equivalently analysed either in terms of Fourier spectra or through measurements of autocorrelation functions. While the former are more directly related to the actual features of the signals, the latter yield more direct information about the intimate nature of the underlying stochastic process.

\section{Modelling noise processes}

Models of noise processes can derive either from assumptions directly concerning the average time course of the noise signals or from the characterization of the probability space of the underlying stochastic process. One approach leads directly to the evaluation of time averages such as autocorrelation functions or power spectra. The other deals with expectation values, typically the covariance, of the stochastic observable. For ergodic processes the two approaches are, in principle, completely equivalent. In practical cases, however, one may be far more convenient than the other.

Thus, it may occur that the time course of the observed variable can be adequately described as the superposition of many random and independent elementary contributions each constituting a rare event of well defined shape. The fluctuations of such variables are better characterized directly in terms of their power spectrum, which is related in a very simple way to that of each elementary contribution (Campbell's theorem; see Rice, 1944).

All models for shot effect noise processes follow this approach (for an exhaustive review of such models see Verveen \& De Felice, 1974). The most classical type of such processes is the shot noise in vacuum tubes due to the random extraction of electrons from the cathode. The stochastic nature of these phenomena is mainly confined to the triggering 
of each elementary waveform, further randomness being possibly introduced when the shot events, rather than being of fixed shape, are allowed to have statistical distributions of, e.g. amplitude and duration.

In contrast with the above type of random processes, one may deal with a stochastic observable, $X$, which is more conveniently described in terms of its probability densities, $f_{n}\left[\left(x_{1}, t_{1}\right) \ldots\left(x_{n}, t_{n}\right)\right]$. This is certainly the case when $X$ can be assumed to be markovian, namely satisfying the condition:

$$
\begin{aligned}
& f_{n}\left[\left(x_{1}, t_{1}\right),\left(x_{2}, t_{2}\right) \ldots\left(x_{n}, t_{n}\right)\right] \\
& \quad=f_{1}\left(x_{1}, t_{1}\right) f_{2}\left[\left(x_{2}, t_{2}-t_{1}\right) /\left(x_{1}, \circ\right)\right] \ldots f_{2}\left[\left(x_{n}, t_{n}-t_{n-1}\right) /\left(x_{n-1}, \circ\right)\right] .
\end{aligned}
$$

Equation (A 22) merely implies that the probability of future values of $X$ depends only on its present value and not on its past time evolution. Equation (A 22) has the far reaching consequence that the observable $X$ is completely specified (under stationary conditions) solely by the conditional probability density $f_{2}\left[(x, t) /\left(x_{0}, 0\right)\right]$. The theory of channel noise discussed in the text is concerned with a particular case of (discrete) markovian variable.

\section{Common sources of electrical noise}

At least three sources of random fluctuations are generally encountered when measuring any electrical parameter in physical systems: thermal noise, shot noise, I/f (flicker) noise. The main characteristics of these electrical noise sources are briefly summarized below. Detailed discussions can be found in many books or reviews (e.g. Van der Ziel, 1970; Davenport \& Root, 1958; Verveen \& De Felice, 1974).

Thermal noise. This is the first type of electrical noise to have been actually measured experimentally (Johnson, I928). According to Nyquist theorem (Nyquist, I928) such noise is a universal characteristic of any equilibrium thermodynamic system containing charged particles. The voltage fluctuations across any two points of a system in thermal equilibrium have a power spectrum given by:

$$
S_{V}(f)={ }_{4 k T} \operatorname{Re} Z,
$$

where $k$ is the Boltzman constant, $T$ is the absolute temperature and $\operatorname{Re} Z$ stands for the real part of the system electrical impedance across the two measurement points. According to (A 23) the classical thermal 
noise across a pure ohmic resistance has a constant power spectrum (white noise). However, the quantum mechanical generalization of the Nyquist theorem leads to a spectral density which tends to zero at frequencies of the order of $\mathrm{k} T / h$, where $h$ is the Planck constant (see, e.g. Van der Ziel, 1970). Nyquist theorem can be generalized to systems in steady state or generally in quasi-equilibrium (Lax, 1960), although other noise sources may also appear in such situations. It can also be extended to encompass the fluctuations around equilibrium, or quasi equilibrium, of any physical observable, other than electrical in origin. It then relates the appropriate general admittance of the system to the power spectrum of the fluctuations of the observable. It is practically equivalent to the fluctuation-dissipation theorem (Kubo, 1957; Kubo et al. 1973; Lax, 1960).

Shot-noise. Whenever an average electric current, $\bar{I}$, flows between any two points of a system, this is no longer in equilibrium. In addition to the thermal noise other noise sources may appear. The classical shot noise is shown by those systems in which the charged carriers of the electrical current, each bearing a charge which is a multiple integer of the electronic charge, $e$, move independently from each other. For electrons in vacuum tubes or solid-state junctions, the current shotnoise has a power spectral density given by:

$$
S_{I}(f)=2 e \bar{I}
$$

yielding a white noise up to very high frequencies. For $f>\sim \mathrm{r} / \tau$, where $\tau$ is of the order of the electrons transit time, $S_{I}(f)$ declines to zero in various ways depending on the time characteristics of the single 'shot' current event (Van der Ziel, 1970; Davenport \& Root, 1958). Shot noise-like phenomena are expected to occur in any system where the current is carried by non-interacting, or moderately interacting carriers.

I/f noise. Current, and/or voltage, fluctuations with power spectral densities which vary in a wide range of frequencies as $f^{-\alpha}$, where $\alpha$ is close to unity, have been described in many systems. Being in excess to the theoretical Nyquist noise, these phenomena can occur only in non-equilibrium systems. Recent experimental work, mostly prompted by the finding of a large $\mathrm{I} / \mathrm{f}$ noise contribution in the electrical fluctuations of nerve membranes, has shown that indeed $I / f$ noise is a quite general phenomenon affecting many systems away from thermodynamic 
equilibrium. Thus, $\mathrm{x} / f$ noise is found in artificial membranes (De Felice \& Michalides, 1972; Michalides et al. 1973), in microelectrodes (De Felice \& Firth, 1970), or across small holes separating two different electrolytic solutions (Hooge \& Gaal, 1970). A general interpretation of the origin of $\mathrm{I} / \mathrm{f}$ noise is still lacking, despite the fact that many particular models can be shown to lead to $\mathrm{I} / f$ noise expectations. An exhaustive review of theoretical and experimental studies on $\mathbf{I} / f$ noise in a large variety of systems is given by Verveen \& De Felice (1974).

\section{REFERENCES}

Alvarez, O., Latorre, R. \& Vergudo, P. (I975). Kinetic characteristics of the EIM channel in oxidized cholesterol and brain lipid bilayer membranes. F. gen. Physiol. 65, 421-39.

ANDerson, C. R. \& STevens, C. F. (I973). Voltage clamp analysis of acetylcholine produced end-plate current fluctuations at frog neuromuscular junction. $\mathcal{F}$. Physiol., Lond. 235, 655-91.

Armstrong, C. M. (1975). Ionic pores, gates, and gating currents. $Q$. Rev. Biophys. 7, 179-210.

Armstrong, C. M. \& Bezanilla, F. (1974). Charge movement associated with the opening and closing of the activation gates of the Na channels. f. gen. Physiol. 63, 533-52.

Armstrong, C. M. \& Binstock, L. (1965). Anomalous rectification in the squid giant axon injected wth tetraethylammonium chloride. $\mathcal{F}$. gen. Physiol. 48, 859-72.

BAmberG, E. \& LÄUgER, P. (1973). Channel formation kinetics of gramicidin A in lipid bilayer membranes. F. Membrane Biol. II, 177-94.

BEAN, R. C. (1972). Multiple conductance states in single channels of variable resistance in lipid bilayer membranes. $\mathcal{F}$. Membrane Biol. 7, I 5-28.

Bean, R. C., Shepherd, W. C., Chan, H. \& Eichner, J. T. (I969). Discrete conductance fluctuations in lipid bilayer protein membranes. $\mathcal{F}$. gen. Physiol. 53, 741-575.

Begenisich, T. \& SteVEns, C. F. (1975). How many conductance states do potassium channels have? Biophys. 7 . 15, 843-46.

BoHEIM, G. (1974). Statistical analysis of alamethicin channels in black lipid membranes. F. Membrane Biol. 19, 277-302.

Cass, A., Finkelstein, A. \& KresPi, V. (1970). The ion permeability induced in thin lipid membranes by the polyene antibiotics nystatin and amphotericin B. F. gen. Physiol. 56, 100-23.

Chen, D. R. \& HILl, H. T. (1973). Fluctuations and noise in kinetic system: application to $\mathrm{K}^{+}$channels in the squid axon. Biophys. $7 . \mathbf{1 3}$, I $276-95$.

CoLE, K. S. (1968). Membranes, Ions and Impulses. Berkeley: University of California Press. 
Cole, K. S. \& Moore, J. W. (1960). Potassium ion current in the squid giant axon: dynamic characteristic. Biophys. $\mathcal{F}$. 1, I-14.

ConTI, F. (1970). Nerve membrane electrical characteristics near the resting state. Biophysik 6, 257-70.

Conti, F., De Felice, L. J. \& Wanke, E. (1975). Potassium and sodium ion current noise in the membrane of the squid giant axon. $\mathcal{F}$. Physiol., Lond. 248, 45-82.

Conti, F., Fioravanti, R. \& Wanke, E. (1973). Fenomeni di breakdown elettrico nella membrana dell'assone gigante di calamaro. Atti del I Congresso della Società Italiana di Biofisica Pura ed Applicata.

Davenport, W. B. \& Root, W. L. (1958). An Introduction to the Theory of Random Signals and Noise. New York: McGraw-Hill Book Co.

De FeliCE, L. J. \& FIRTH, D. R. (I970). Spontaneous voltage fluctuations in glass microelectrodes. IEEE Trans. Bio-med. Engng 18, 339-5I.

De Felice, L. J. \& Michalides, J. P. L. M. (1972). Electrical noise from synthetic membranes. F. Membrane Biol. 9, 26I-9o.

De Felice, L. J., Wanke, E. \& ConTi, F. (r975). Potassium and sodium current noise from squid axon membranes. Fedn Proc. Fedn Am. Socs exp. Biol. 34, 1338-42.

Del Castillo, J. \& Katz, B. (1954). Changes in end-plate activity produced by pre-synaptic depolarization. F. Physiol., Lond. 124, 586-604.

Derksen, H. E. (1965). Axon membrane voltage fluctuations. Acta physiol. Pharmacol. néerl. 13, 373-466.

Derksen, H. E. \& Verveen, A. A. (1966). Fluctuations of resting membrane potential. Science, N.Y. 151, 1388-9.

Ehrenstein, G., Blumenthal, R., LatorRe, R. \& Lecar, H. (1974). Kinetics of opening and closing of individual EIM channels in lipid bilayer. 7. gen. Physiol. 63, 707-21.

EHRENSTEIN, G. \& GILBERT, D. L. (1966). Slow changes of potassium permeability in the squid giant axon. Biophys. F. 6, 553-64.

Ehrenstein, G., Lecar, H. \& Nossal, R. (1970). The nature of the negative resistance in bimolecular lipid membranes containing excitabilityinducing material. F. gen. Physiol. 65, 1 19-33.

Eisenberg, M., Hall, J.E. \& Mead, C. A. (1973). The nature of the voltage-dependent conductance induced by alamethicin in black lipid membranes. $\mathcal{F}$. Membrane Biol. 14, 143-76.

Feher, G. \& WeISSMan, M. (1973). Fluctuation spectroscopy: determination of chemical reaction kinetics from the frequency spectrum of fluctuations. Proc. natn. Acad. Sci. U.S.A. 70, 870-5.

Fetriplace, R., Gordon, L. G. M., Hladky, S. B., Requena, J., Ringsheim, H. P. \& Haydon, D. A. (1975). In Methods in Membrane Biology, vol. 4 (ed. E. D. Korn). New York and London: Plenum Press.

Finkelstein, A. \& Holz, R. (1972). Acqueous pores created in thin lipid membranes by the polyene antibiotics nystatin and amphotericin $B$. Membranes: A Series of Advances (ed. G. Eisenman). New York: M. Dekker, Inc. 
Fishman, H. M. (1973). Relaxation spectra of potassium channel noise from squid axon membranes. Proc. natn. Acad. Sci. U.S.A. 70, 876-9.

Fishman, H. M. (1975). Noise measurements in axon membranes. Fedn Proc. Fedn Am. Socs exp. Biol. 34, I330-7.

Frankenhaeuser, B. \& Hodgkin, A. L. (I956). The after-effects of impulses in the giant nerve fibers of Loligo. F. Physiol., Lond. 131, 341-76.

Gordon, L. G. M. \& HAYDON, D. A. (1972). The unit conductance channel of alamethicin. Biochim. biophys. Acta 255, 1014-18.

Gordon, M. G. M. \& Haydon, D. A. (1975). Potential-dependent conductances in lipid membranes containing alamethicin. Phil. Trans. $R$. Soc. Lond. B 270, 433-47.

Haydon, D. A., Hladky, S. B. \& Gordon, L. G. M. (1972). The single channel technique in the study of ion transport across membranes. Proc. VIII FEBS Meeting, vol. 28, 307-16.

Hill, T. L. \& Chen YI-DeR (I972a). On the theory of ion transport across the nerve membrane. IV. Noise from the open-close kinetics of $\mathrm{K}^{+}$ channels. Biophys. F. 12, 948-59.

Hill, T. L. \& CHEN YI-DeR (I972b). On the theory of ion transport across the nerve membrane. V. Two models for the Cole-Moore $\mathrm{K}^{+}$hyperpolarization delay. Biophys. F. 12, 960-76.

HLADKY, S. B. \& HaYdon, D. A. (1970). Discreteness of conductance change in bimolecular lipid membranes in the presence of certain antibiotics. Nature, Lond. 225, $45^{\mathrm{I}-3}$.

Hladky, S. B. \& Haydon, D. A. (1972). Ion transfer across lipid membranes in the presence of gramicidin A. I. Studies of the unit conductance channel. Biochim. biophys. Acta, 274, 294-312.

Hodgrin, H. L. \& HUXLEY, A. F. (I952). A quantitative description of membrane current and its application to conduction and excitation in nerve. F. Physiol., Lond. II7, 507-44.

HoOgE, F. N. \& GAAL, J. L. M. (I970). Fluctuations with a I/f spectrum in the conductance of ionic solutions and in the voltage of concentration cells. Philips Res. Rep. 26, 77-90.

Johnson, J. B. (1928). Thermal agitation of electricity in conductors. Phys. Rev. 32, 97-ro9.

KAC, M. (1947). Random walk and the theory of Brownian motion. Am. Math. Monthly 54, 369-91. Also reprinted in Selected Papers on Noise and Stochastic Processes (ed. N. Wax). New York: Dover Publ. Inc., I954.

KATZ, B. \& MilEDI, R. (1970). Membrane noise produced by acetylcholine. Nature, Lond. 225, 962-3.

KATZ, B. \& MiledI, R. (1972). The statistical nature of the acetylcholine potential and its molecular components. F. Physiol., Lond. 224, 665-99.

KatZ, B. \& MileDI, R. (1973). The characteristics of 'end-plate noise' produced by different depolarizing drugs. $\mathcal{F}$. Physiol., Lond. 230, 707-1 7.

KEYNES, R. D. (1975). The organization of the ionic channels in nerve membranes. NINDS 25th Anniversary volume. 
Keynes, R. D., Bezanilla, F., Rojas, E. \& Taylor, R. E. (I975). The rate of action of tetrodotoxin on sodium conductance in the squid giant axon. Phil. Trans. R. Soc. Lond. B 270, 365-75.

KeYnes, R. D. \& RojAs, E. (1976). The temporal and steady-state relationships between activation of the sodium conductance and movement of the gating particles in the squid giant axon. F. Physiol., Lond. 255, 157-189.

KhINCHIN, A. I. (1949). Mathematical Foundations of Statistical Mechanics. New York: Dover Publ. Inc.

KolB, H. A., LÄUger, P. \& BAMBERG, E. (1975). Correlation analysis of electrical noise in lipid bilayer membranes: Kinetics of gramicidin A channels. f. Membrane Biol. 20, I33-54.

Kuвo, R. (1957). Statistical mechanical theory of irreversible processes. General theory and simple applications to magnetic and conduction problems. F. Physiol. Soc. Japan 12, 570-86.

Kubo, R., Matsuo, K. \& Kazuhiro, K. (1973). Fluctuation and relaxation of macrovariables. F. Stat. Phys. 9, 51-96.

LATORRE, R., Ehrenstein, G. \& LeCAR, H. (I972). Ion transport through excitability-inducing material (EIM) channels in lipid bilayer membranes. F. gen. Physiol. 6o, 72-85.

LäUGER, P. (1972). Carrier mediated ion transport. Science, N.Y. 178, 24-30.

LAX, M. (1960). Fluctuations from the nonequilibrium steady state. Rev. mod. Phys. 32, 25-64.

LeCAR, H. \& Nossal, R. (1971). Theory of threshold fluctuations in nerves I, II. Biophys. F. II, 1048-84.

LEE, Y. W. (1960). Statistical Theory of Communication. New York: J. Wiley and Sons.

Levinson, S. R. \& Meves, H. (1975). The binding of tritiated tetrodoxin to squid giant axons. Phil. Trans. R. Soc. Lond. B 270, 349-52.

Mauro, A., Nanavati, R. P. \& Heyer, E. (1972). Time-variant conductance of bilayer membranes treated with monazomycin and alamethicin. Proc. natn. Acad. Sci. U.S.A. 69, 3742-4.

Michalides, J. P. L. M., Wallaakt, R. A. M. \& De Felice, L. J. (1973). Electrical noise from PVC-Membranes. Pflïgers Arch. 34I, 97-104.

Middleton, D. (I960). An Introduction to Statistical Communication Theory. New York: McGraw-Hill.

Motchenbauer, C. D. \& Fitchen, F. C. (1973). Low-noise Electronic Design, $35^{8} \mathrm{pp}$. New York: Wiley-Interscience.

MuelleR, P. \& Rudin, D. O. ( 1963$)$. Induced excitability in reconstituted cell membrane structure. F. theor. Biol. 4, 268-280.

Mueller, P., Rudin, D. O., Tien, H.T. \& WestcotT, W. W. (1962). Reconstitution of all membrane structure in vitro and its transformation into an excitable system. Nature, Lond. 194, 979-81.

MulleR, R. U. \& Finkelstein, A. (1972a). Voltage-dependent conductance induced in thin lipid membranes by monazomycin. F. gen. Physiol. 6o, 263-84.

MUlleR, R. U. \& Finkelstein, A. (1972b). The effect of surface charge on 
the voltage-dependent conductance induced in thin lipid membranes by monazomycin. F. gen. Physiol. 60, 285-306.

Mullins, L. J. (1959). An analysis of conductance changes in squid axon. f. gen. Physiol. 42, IOI3-35.

Mullins, L. J. (1968). A single channel or a dual channel mechanism for nerve excitation. F. gen. Physiol. 52, 550-3.

Narahashi, T., Moore, J. W. \& ScotT, W. R. (1964). Tetrodotoxin blockage of sodium conductance increase in lobster giant axons. F. gen. Physiol. 47, $965-74$.

NeHER, E. \& ZingsheIM, H. P. (1974). The properties of ionic channels measured by noise analysis in this lipid membranes. Pflügers Arch. ges. Physiol. 351, 6I-7.

NonNer, W., Rojas, E. \& Stämpfli, R. (I975). Gating currents in the node of Ranvier: voltage and time dependence. Phil. Trans. R. Soc. Lond. B 270, 483-92.

NYquist, H. (1928). Thermal agitation of electric charge in conductors. Phys. Rev. 32, I 10-13.

Papoulis, A. (1965). Probability, Random Variables, and Stochastic Processes. New York: McGraw-Hill Book Co.

POOLER, J. P. \& OXFord, G. S. (1972). Low membrane resistance in sucrose gap - a parallel leakage path. Biochim. biophys. Acta 255, 68I-4.

PousSART, D. J. M. (197I). Membrane current noise in lobster axon under voltage clamp. Biophys. F. II, 2II-34.

PousSaRT, D. J. M. (1973). Low-level average power measurements: noise figure improvement through parallel or series connection of noisy amplifiers. Rev. scient. Instrum. 44, 1049-52.

Rabiner, L. R. \& Rader, C. M. (1972). Digital Signal Processing, 518 pp. New York: IEEE Press.

RICE, S. O. (1944). Mathematical analysis of random noise. Bell System Techn. F. 23, 282-304. Also reprinted in Selected Papers on Noise and Stochastic Processes (ed. N. Wax). New York: Dover Publ. Inc., 1954.

RojAS, E. \& KeYNes, R. D. (1975). On the relation between displacement currents and activation of the sodium conductance in the squid giant axon. Phil. Trans. R. Soc. Lond. B 270, 459-82.

SEGAL, J. R. (1972). Electrical fluctuations associated with active transport. Biophys. f. 12, 1371-9o.

Siebenga, E., De Goede, J. \& Verveen, A. A. (r974). The influence of TTX, DNP and TEA on membrane flicker noise and shot effect noise of the frog node of Ranvier. Pfügers Arch. ges. Physiol. 351, 25-34.

Siebenga, E., Meyer, A. \& Verveen, A. A. (I973). Membrane shot noise in electrically depolarized nodes of Ranvier. Pflügers Arch. ges. Physiol. 341, 97-104.

Siebenga, E. \& VerveEn, A. A. (197I). The dependence of the $I / f$ noise intensity of the node of Ranvier on membrane potential. In Proc. Ist Eur. Biophys. Congress (eds. A. Springer, Locker, and H. Lederer), pp. 219-23, Vienna: Verlag Wiener Medizinischen Chem. Akad. 
Siebenga, E. \& Verveen, A. A. (1972). Membrane noise and ion transport in the node of Ranvier. In Passive Permeability of Cell Membranes, Biomembranes, vol. 3 (eds. J. F. Slegers and F. Kreuzer). New York: Plenum Publ. Co.

Siebenga, E. \& VerveEN, A. A. (I970). Noise voltage of axonal membrane. Pflügers Arch. ges. Physiol. 318, 267.

Stevens, F. (1972). Inferences about membrane properties from electrical noise measurements. Biophys. F. 12, 1028-47.

TASAKI, I. \& Hagiwara, S. (I957). Demonstration of two stable potential states in squid giant axon under tetraethylammonium chloride. $\mathcal{F}$ gen. Physiol. 40, $85 \mathrm{I}-85$.

UHLENBECK, G. E. \& ORNSTEIN, L. S. (I930). On the theory of Brownian motion. Phys. Rev. 36, 823-841. Also reprinted in Selected Papers on Noise and Stochastic Processes (ed. N. Wax). New York: Dover Publ. Inc., 1954 .

URRY, D. W. (1971). The gramicidin A transmembrane channel: a proposed $\pi_{(\mathrm{L}, \mathrm{D})}$ helix. Proc. natn. Acad. Sci. U.S.A. 68, 672-6.

URry, D. W., Goodall, M. C., Glickson, J. D. \& MeYers, D. F. (197I). The gramicidin A transmembrane channel: characteristics of head-tohead dimerized $\pi_{(\mathrm{L}, \mathrm{D})}$ helices. Proc. natn. Acad. Sci. U.S.A. 68, 1907-I I.

VAN Den Berg, R. J. (I975). Conductance fluctuation in Ranvier nodes.

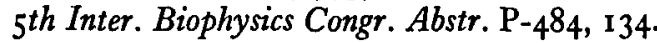

VAN DER ZIEL, A. (1970). Noise: Sources, Characterization, Measurements. Engelwood Cliffs, N. Y.: Prentice-Hall. Inc.

Van Driessche \& Borghgraef, R. (I975). Noise generated during ion transport across frog skin. Archs int. Physiol. Biochim. 83, 140-3.

Verveen, A. A. \& De Felice, L. J. (1974). Membrane noise. Prog. Biophys. molec. Biol. 28, 189-265.

VerveEn, A. A. \& Derksen, H. H. (1965). Fluctuations in membrane potential of axons and the problem of coding. Kybernetik 2, 152-60.

Verveen, A. A. \& DerkseN, H. E. (1968). Fluctuation phenomena in nerve membrane. Proc. IEEE 56, 906-16.

VerveEn, A. A. \& Derksen, H. E. (I969). Amplitude distribution of axon membrane noise voltage. Acta physiol. pharmacol. néerl. 15, 353-79.

WANKE, E. (1975). Monazomycin and nystatin channel noise. 5th Inter. Biophys. Congr. Abstr. P-368, I 12.

Wanke, E., De Frlice, L. J. \& Conti, F. (I974). Voltage noise and current noise in space clamped squid giant axon. Pfiügers Arch. ges. Physiol. 347, $63-74$.

Yafuso, M., Kennedy, S. J. \& Freeman, A. R. (1974). Spontaneous conductance changes, multilevel conductance states and negative differential resistance in oxidized cholesterol black lipid membranes. $\mathcal{F}$. Membrane Biol. I7, $201-12$.

ZINGSheIm, H. P. \& NeHER, E. (1974). The equivalence of fluctuation analysis and chemical relaxation measurements: a study of ion pore formation in thin lipid membranes. Biophys. Chem. 2, 197-207. 